Marquette University

e-Publications@Marquette

Physics Faculty Research and Publications

Physics, Department of

6-1-2017

\title{
The IceCube Realtime Alert System
}

Karen Andeen

Marquette University, karen.andeen@marquette.edu

IceCube Collaboration

Accepted version. Astroparticle Physics, Vol. 92 (June 2017): 30-41. DOI. C 2017 Elsevier B.V. Used with permission.

A complete list of authors available in the article text. 
Marquette University

e-Publications@Marquette

\section{Physics Faculty Research and Publications/College of Arts and Sciences}

This paper is NOT THE PUBLISHED VERSION; but the author's final, peer-reviewed manuscript. The published version may be accessed by following the link in the citation below.

Astroparticle Physics, Vol. 92, No. 3 (June, 2017). DOI. This article is C Elsevier and permission has been granted for this version to appear in e-Publications@Marquette. Elsevier does not grant permission for this article to be further copied/distributed or hosted elsewhere without the express permission from Elsevier.

\section{Contents}

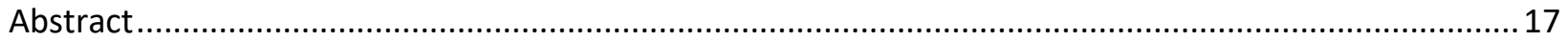

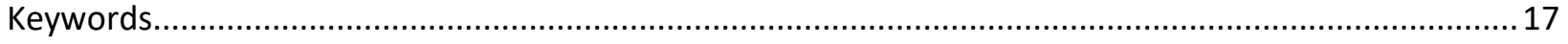

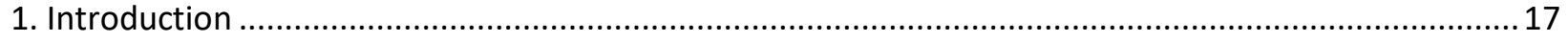

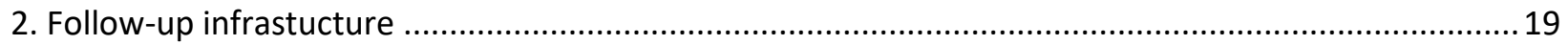

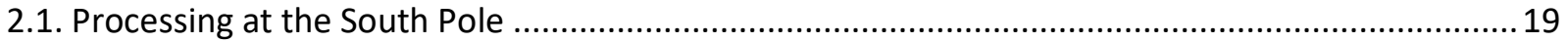

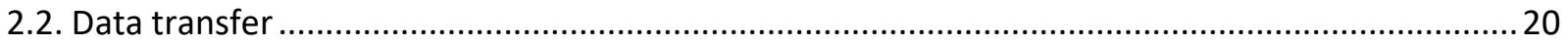

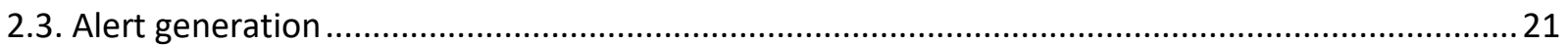

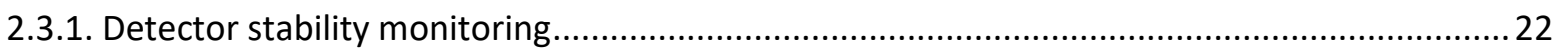

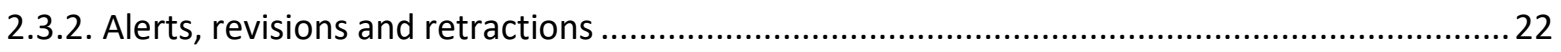

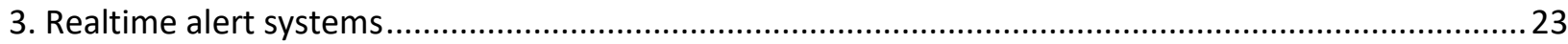

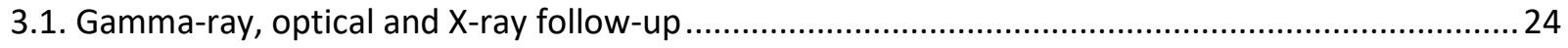

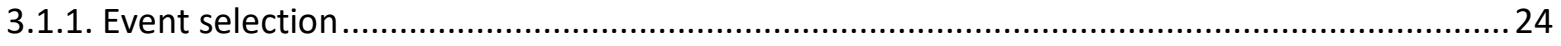

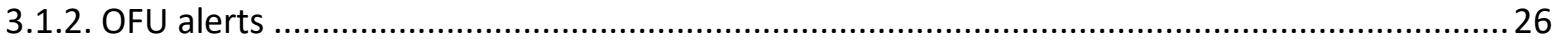

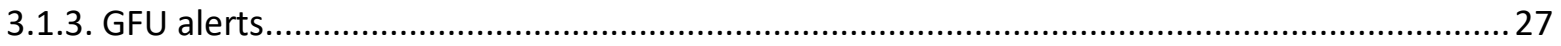

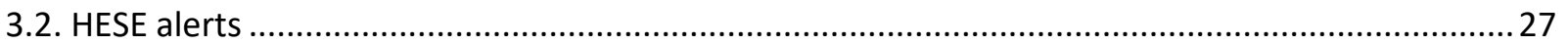

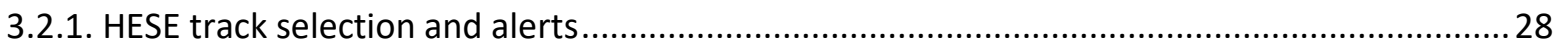

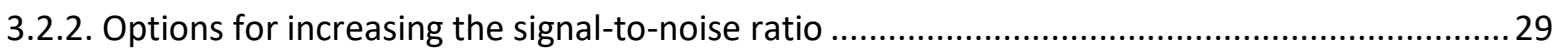




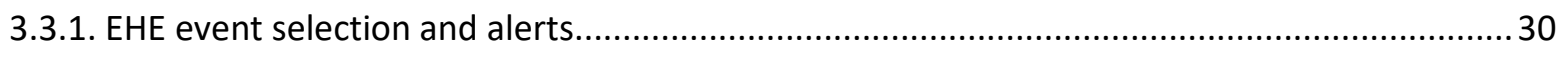

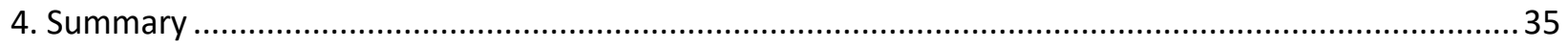

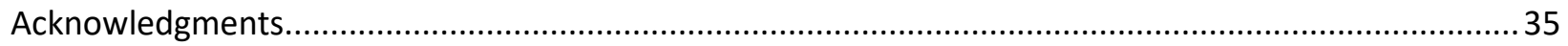

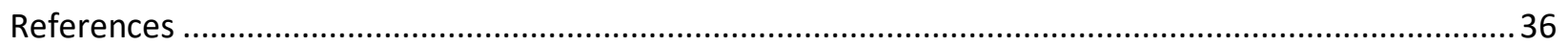

\title{
The IceCube Realtime Alert System
}

\author{
M.G. Aartsen \\ Department of Physics, University of Adelaide, Adelaide, 5005, Australia \\ M. Ackermann \\ DESY, D-15735 Zeuthen, Germany \\ J. Adams \\ Department of Physics and Astronomy, University of Canterbury, Private Bag 4800, Christchurch, New \\ Zealand \\ J.A. Aguilar \\ Université Libre de Bruxelles, Science Faculty CP230, B-1050 Brussels, Belgium \\ M. Ahlers \\ Department of Physics and Wisconsin IceCube Particle Astrophysics Center, University of Wisconsin, Madison, WI \\ 53706, USA \\ M. Ahrens \\ Oskar Klein Centre and Department of Physics, Stockholm University, SE-10691 Stockholm, Sweden \\ D. Altmann \\ Erlangen Centre for Astroparticle Physics, Friedrich-Alexander-Universität Erlangen-Nürnberg, D-91058 Erlangen, \\ Germany \\ K. Andeen \\ Department of Physics, Marquette University, Milwaukee, WI, 53201, USA \\ T. Anderson \\ Department of Physics, Pennsylvania State University, University Park, PA 16802, USA \\ I. Ansseau \\ Université Libre de Bruxelles, Science Faculty CP230, B-1050 Brussels, Belgium \\ G. Anton \\ Erlangen Centre for Astroparticle Physics, Friedrich-Alexander-Universität Erlangen-Nürnberg, D-91058 Erlangen, \\ Germany \\ M. Archinger \\ Institute of Physics, University of Mainz, Staudinger Weg 7, D-55099 Mainz, Germany \\ C. Argüelles \\ Department of Physics, Massachusetts Institute of Technology, Cambridge, MA 02139, USA
}




\section{J. Auffenberg}

III. Physikalisches Institut, RWTH Aachen University, D-52056 Aachen, Germany

S. Axani

Department of Physics, Massachusetts Institute of Technology, Cambridge, MA 02139, USA

X. Bai

Physics Department, South Dakota School of Mines and Technology, Rapid City, SD 57701, USA

S.W. Barwick

Department of Physics and Astronomy, University of California, Irvine, CA 92697, USA

V. Baum

Institute of Physics, University of Mainz, Staudinger Weg 7, D-55099 Mainz, Germany

R. Bay

Department of Physics, University of California, Berkeley, CA 94720, USA

J.J. Beatty

Department of Physics and Center for Cosmology and Astro-Particle Physics, Ohio State University, $\mathrm{Columbus,} \mathrm{OH}$ 43210, USA

\section{J. Becker Tjus}

Fakultät für Physik \& Astronomie, Ruhr-Universität Bochum, D-44780 Bochum, Germany

K.-H. Becker

Department of Physics, University of Wuppertal, D-42119 Wuppertal, Germany

S. BenZvi

Department of Physics and Astronomy, University of Rochester, Rochester, NY 14627, USA

D. Berley

Department of Physics, University of Maryland, College Park, MD 20742, USA

E. Bernardini

DESY, D-15735 Zeuthen, Germany

A. Bernhard

Physik-department, Technische Universität München, D-85748 Garching, Germany

D.Z. Besson

Department of Physics and Astronomy, University of Kansas, Lawrence, KS 66045, USA

G. Binder

Lawrence Berkeley National Laboratory, Berkeley, CA 94720, USA

Department of Physics, University of California, Berkeley, CA 94720, USA

D. Bindig

Department of Physics, University of Wuppertal, D-42119 Wuppertal, Germany

M. Bissok

III. Physikalisches Institut, RWTH Aachen University, D-52056 Aachen, Germany

E. Blaufuss

Department of Physics, University of Maryland, College Park, MD 20742, USA

S. Blot

DESY, D-15735 Zeuthen, Germany

C. Bohm

Oskar Klein Centre and Department of Physics, Stockholm University, SE-10691 Stockholm, Sweden

M. Börner

Department of Physics, TU Dortmund University, D-44221 Dortmund, Germany 


\section{F. Bos}

Fakultät für Physik \& Astronomie, Ruhr-Universität Bochum, D-44780 Bochum, Germany

D. Bose

Department of Physics, Sungkyunkwan University, Suwon 440-746, Korea

S. Böser

Institute of Physics, University of Mainz, Staudinger Weg 7, D-55099 Mainz, Germany

O. Botner

Department of Physics and Astronomy, Uppsala University, Box 516, S-75120 Uppsala, Sweden

J. Braun

Department of Physics and Wisconsin IceCube Particle Astrophysics Center, University of Wisconsin, Madison, WI 53706, USA

L. Brayeur

Vrije Universiteit Brussel (VUB), Dienst ELEM, B-1050 Brussels, Belgium

H.-P. Bretz

DESY, D-15735 Zeuthen, Germany

S. Bron

Département de physique nucléaire et corpusculaire, Université de Genève, $\mathrm{CH}-1211$ Genève, Switzerland

A. Burgman

Department of Physics and Astronomy, Uppsala University, Box 516, S-75120 Uppsala, Sweden

T. Carver

Département de physique nucléaire et corpusculaire, Université de Genève, $\mathrm{CH}-1211$ Genève, Switzerland

M. Casier

Vrije Universiteit Brussel (VUB), Dienst ELEM, B-1050 Brussels, Belgium

E. Cheung

Department of Physics, University of Maryland, College Park, MD 20742, USA

D. Chirkin

Department of Physics and Wisconsin IceCube Particle Astrophysics Center, University of Wisconsin, Madison, WI 53706, USA

\section{A. Christov}

Département de physique nucléaire et corpusculaire, Université de Genève, $\mathrm{CH}-1211$ Genève, Switzerland

\section{K. Clark}

Department of Physics, University of Toronto, Toronto, Ontario, M5S 1A7, Canada

L. Classen

Institut für Kernphysik, Westfälische Wilhelms-Universität Münster, D-48149 Münster, Germany

S. Coenders

Physik-department, Technische Universität München, D-85748 Garching, Germany

G.H. Collin

Department of Physics, Massachusetts Institute of Technology, Cambridge, MA 02139, USA

J.M. Conrad

Department of Physics, Massachusetts Institute of Technology, Cambridge, MA 02139, USA

D.F. Cowen

Department of Astronomy and Astrophysics, Pennsylvania State University, University Park, PA 16802, USA

Department of Physics, Pennsylvania State University, University Park, PA 16802, USA

\section{R. Cross}


Department of Physics and Astronomy, University of Rochester, Rochester, NY 14627, USA

M. Day

Department of Physics and Wisconsin IceCube Particle Astrophysics Center, University of Wisconsin, Madison, WI 53706, USA

J.P.A.M. de André

Department of Physics and Astronomy, Michigan State University, East Lansing, MI 48824, USA

C. De Clercq

Vrije Universiteit Brussel (VUB), Dienst ELEM, B-1050 Brussels, Belgium

E. del Pino Rosendo

Institute of Physics, University of Mainz, Staudinger Weg 7, D-55099 Mainz, Germany

H. Dembinski

Bartol Research Institute and Department of Physics and Astronomy, University of Delaware, Newark, DE 19716, USA

S. De Ridder

Department of Physics and Astronomy, University of Gent, B-9000 Gent, Belgium

P. Desiati

Department of Physics and Wisconsin IceCube Particle Astrophysics Center, University of Wisconsin, Madison, WI 53706, USA

K.D. de Vries

Vrije Universiteit Brussel (VUB), Dienst ELEM, B-1050 Brussels, Belgium

G. de Wasseige

Vrije Universiteit Brussel (VUB), Dienst ELEM, B-1050 Brussels, Belgium

M. de With

Institut für Physik, Humboldt-Universität zu Berlin, D-12489 Berlin, Germany

T. DeYoung

Department of Physics and Astronomy, Michigan State University, East Lansing, MI 48824, USA

J.C. Díaz-Vélez

Department of Physics and Wisconsin IceCube Particle Astrophysics Center, University of Wisconsin, Madison, WI 53706, USA

V. di Lorenzo

Institute of Physics, University of Mainz, Staudinger Weg 7, D-55099 Mainz, Germany

H. Dujmovic

Department of Physics, Sungkyunkwan University, Suwon 440-746, Korea

J.P. Dumm

Oskar Klein Centre and Department of Physics, Stockholm University, SE-10691 Stockholm, Sweden

M. Dunkman

Department of Physics, Pennsylvania State University, University Park, PA 16802, USA

B. Eberhardt

Institute of Physics, University of Mainz, Staudinger Weg 7, D-55099 Mainz, Germany

T. Ehrhardt

Institute of Physics, University of Mainz, Staudinger Weg 7, D-55099 Mainz, Germany

B. Eichmann

Fakultät für Physik \& Astronomie, Ruhr-Universität Bochum, D-44780 Bochum, Germany

P. Eller

Department of Physics, Pennsylvania State University, University Park, PA 16802, USA 


\section{S. Euler}

Department of Physics and Astronomy, Uppsala University, Box 516, S-75120 Uppsala, Sweden

\section{P.A. Evenson}

Bartol Research Institute and Department of Physics and Astronomy, University of Delaware, Newark, DE 19716, USA

\section{S. Fahey}

Department of Physics and Wisconsin IceCube Particle Astrophysics Center, University of Wisconsin, Madison, WI 53706, USA

A.R. Fazely

Department of Physics, Southern University, Baton Rouge, LA 70813, USA

A.R. Fazely

Department of Physics, Southern University, Baton Rouge, LA 70813, USA

J. Feintzeig

Department of Physics and Wisconsin IceCube Particle Astrophysics Center, University of Wisconsin, Madison, WI 53706, USA

J. Felde

Department of Physics, University of Maryland, College Park, MD 20742, USA

K. Filimonov

Department of Physics, University of California, Berkeley, CA 94720, USA

C. Finley

Oskar Klein Centre and Department of Physics, Stockholm University, SE-10691 Stockholm, Sweden

S. Flis

Oskar Klein Centre and Department of Physics, Stockholm University, SE-10691 Stockholm, Sweden

\section{C.-C. Fösig}

Institute of Physics, University of Mainz, Staudinger Weg 7, D-55099 Mainz, Germany

\section{A. Franckowiak}

DESY, D-15735 Zeuthen, Germany

E. Friedman

Department of Physics, University of Maryland, College Park, MD 20742, USA

T. Fuchs

Department of Physics, TU Dortmund University, D-44221 Dortmund, Germany

T.K. Gaisser

Bartol Research Institute and Department of Physics and Astronomy, University of Delaware, Newark, DE 19716, USA

J. Gallagher

Department of Astronomy, University of Wisconsin, Madison, WI 53706, USA

L. Gerhardt

Lawrence Berkeley National Laboratory, Berkeley, CA 94720, USA

Department of Physics, University of California, Berkeley, CA 94720, USA

K. Ghorbani

Department of Physics and Wisconsin IceCube Particle Astrophysics Center, University of Wisconsin, Madison, WI 53706, USA

W. Giang

Department of Physics, University of Alberta, Edmonton, Alberta, T6G 2E1, Canada

\section{Gladstone}


Department of Physics and Wisconsin IceCube Particle Astrophysics Center, University of Wisconsin, Madison, WI 53706, USA

T. Glauch

III. Physikalisches Institut, RWTH Aachen University, D-52056 Aachen, Germany

T. Glüsenkamp

Erlangen Centre for Astroparticle Physics, Friedrich-Alexander-Universität Erlangen-Nürnberg, D-91058 Erlangen, Germany

\section{A. Goldschmidt}

Lawrence Berkeley National Laboratory, Berkeley, CA 94720, USA

\section{J.G. Gonzalez}

Bartol Research Institute and Department of Physics and Astronomy, University of Delaware, Newark, DE 19716, USA

D. Grant

Department of Physics, University of Alberta, Edmonton, Alberta, T6G 2E1, Canada

Z. Griffith

Department of Physics and Wisconsin IceCube Particle Astrophysics Center, University of Wisconsin, Madison, WI 53706, USA

C. Haack

III. Physikalisches Institut, RWTH Aachen University, D-52056 Aachen, Germany

A. Hallgren

Department of Physics and Astronomy, Uppsala University, Box 516, S-75120 Uppsala, Sweden

F. Halzen

Department of Physics and Wisconsin IceCube Particle Astrophysics Center, University of Wisconsin, Madison, WI 53706, USA

E. Hansen

Niels Bohr Institute, University of Copenhagen, DK-2100 Copenhagen, Denmark

T. Hansmann

III. Physikalisches Institut, RWTH Aachen University, D-52056 Aachen, Germany

K. Hanson

Department of Physics and Wisconsin IceCube Particle Astrophysics Center, University of Wisconsin, Madison, WI 53706, USA

D. Hebecker

Institut für Physik, Humboldt-Universität zu Berlin, D-12489 Berlin, Germany

D. Heereman

Université Libre de Bruxelles, Science Faculty CP230, B-1050 Brussels, Belgium

K. Helbing

Department of Physics, University of Wuppertal, D-42119 Wuppertal, Germany

R. Hellauer

Department of Physics, University of Maryland, College Park, MD 20742, USA

S. Hickford

Department of Physics, University of Wuppertal, D-42119 Wuppertal, Germany

J. Hignight

Department of Physics and Astronomy, Michigan State University, East Lansing, MI 48824, USA

G.C. Hill

Department of Physics, University of Adelaide, Adelaide, 5005, Australia 


\section{K.D. Hoffman}

Department of Physics, University of Maryland, College Park, MD 20742, USA

\section{K.D. Hoffman}

Department of Physics, University of Maryland, College Park, MD 20742, USA

K. Hoshina

Department of Physics and Wisconsin IceCube Particle Astrophysics Center, University of Wisconsin, Madison, WI 53706, USA

\section{F. Huang}

Department of Physics, Pennsylvania State University, University Park, PA 16802, USA

\section{Huber}

Physik-department, Technische Universität München, D-85748 Garching, Germany

K. Hultqvist

Oskar Klein Centre and Department of Physics, Stockholm University, SE-10691 Stockholm, Sweden

S. In

Department of Physics, Sungkyunkwan University, Suwon 440-746, Korea

A. Ishihara

Department of Physics and Institute for Global Prominent Research, Chiba University, Chiba 263-8522, Japan

E. Jacobi

DESY, D-15735 Zeuthen, Germany

G.S. Japaridze

CTSPS, Clark-Atlanta University, Atlanta, GA 30314, USA

M. Jeong

Department of Physics, Sungkyunkwan University, Suwon 440-746, Korea

K. Jero

Department of Physics and Wisconsin IceCube Particle Astrophysics Center, University of Wisconsin, Madison, WI 53706, USA

B.J.P. Jones

Department of Physics, Massachusetts Institute of Technology, Cambridge, MA 02139, USA

W. Kang

Department of Physics, Sungkyunkwan University, Suwon 440-746, Korea

A. Kappes

Institut für Kernphysik, Westfälische Wilhelms-Universität Münster, D-48149 Münster, Germany

T. Karg

DESY, D-15735 Zeuthen, Germany

A. Karle

Department of Physics and Wisconsin IceCube Particle Astrophysics Center, University of Wisconsin, Madison, WI 53706, USA

U. Katz

Erlangen Centre for Astroparticle Physics, Friedrich-Alexander-Universität Erlangen-Nürnberg, D-91058 Erlangen, Germany

M. Kauer

Department of Physics and Wisconsin IceCube Particle Astrophysics Center, University of Wisconsin, Madison, WI 53706, USA

A. Keivani

Department of Physics, Pennsylvania State University, University Park, PA 16802, USA 


\section{J.L. Kelley}

Department of Physics and Wisconsin IceCube Particle Astrophysics Center, University of Wisconsin, Madison, WI 53706, USA

A. Kheirandish

Department of Physics and Wisconsin IceCube Particle Astrophysics Center, University of Wisconsin, Madison, WI 53706, USA

J. Kim

Department of Physics, Sungkyunkwan University, Suwon 440-746, Korea

M. Kim

Department of Physics, Sungkyunkwan University, Suwon 440-746, Korea

T. Kintscher

DESY, D-15735 Zeuthen, Germany

J. Kiryluk

Department of Physics and Astronomy, Stony Brook University, Stony Brook, NY 11794-3800, USA

T. Kittler

Erlangen Centre for Astroparticle Physics, Friedrich-Alexander-Universität Erlangen-Nürnberg, D-91058 Erlangen, Germany

\section{S.R. Klein}

Lawrence Berkeley National Laboratory, Berkeley, CA 94720, USA

Department of Physics, University of California, Berkeley, CA 94720, USA

G. Kohnen

Université de Mons, 7000 Mons, Belgium

R. Koirala

Bartol Research Institute and Department of Physics and Astronomy, University of Delaware, Newark, DE 19716, USA

H. Kolanoski

Institut für Physik, Humboldt-Universität zu Berlin, D-12489 Berlin, Germany

R. Konietz

III. Physikalisches Institut, RWTH Aachen University, D-52056 Aachen, Germany

L. Köpke

Institute of Physics, University of Mainz, Staudinger Weg 7, D-55099 Mainz, Germany

C. Kopper

Department of Physics, University of Alberta, Edmonton, Alberta, T6G 2E1, Canada

S. Kopper

Department of Physics, University of Wuppertal, D-42119 Wuppertal, Germany

D.J. Koskinen

Niels Bohr Institute, University of Copenhagen, DK-2100 Copenhagen, Denmark

M. Kowalski

Institut für Physik, Humboldt-Universität zu Berlin, D-12489 Berlin, Germany

DESY, D-15735 Zeuthen, Germany

K. Krings

Physik-department, Technische Universität München, D-85748 Garching, Germany

M. Kroll

Fakultät für Physik \& Astronomie, Ruhr-Universität Bochum, D-44780 Bochum, Germany

G. Krückl 
Institute of Physics, University of Mainz, Staudinger Weg 7, D-55099 Mainz, Germany

C. Krüger

Department of Physics and Wisconsin IceCube Particle Astrophysics Center, University of Wisconsin, Madison, WI 53706, USA

J. Kunnen

Vrije Universiteit Brussel (VUB), Dienst ELEM, B-1050 Brussels, Belgium

S. Kunwar

DESY, D-15735 Zeuthen, Germany

N. Kurahashi

Department of Physics, Drexel University, 3141 Chestnut Street, Philadelphia, PA 19104, USA

M. Labare

Department of Physics and Astronomy, University of Gent, B-9000 Gent, Belgium

J.L. Lanfranchi

Department of Physics, Pennsylvania State University, University Park, PA 16802, USA

M.J. Larson

Niels Bohr Institute, University of Copenhagen, DK-2100 Copenhagen, Denmark

F. Lauber

Department of Physics, University of Wuppertal, D-42119 Wuppertal, Germany

D. Lennarz

Department of Physics and Astronomy, Michigan State University, East Lansing, MI 48824, USA

M. Lesiak-Bzdak

Department of Physics and Astronomy, Stony Brook University, Stony Brook, NY 11794-3800, USA

M. Leuermann

III. Physikalisches Institut, RWTH Aachen University, D-52056 Aachen, Germany

L. Lu

Department of Physics and Institute for Global Prominent Research, Chiba University, Chiba 263-8522, Japan

J. Lünemann

Vrije Universiteit Brussel (VUB), Dienst ELEM, B-1050 Brussels, Belgium

J. Madsen

Department of Physics, University of Wisconsin, River Falls, WI 54022, USA

G. Maggi

Vrije Universiteit Brussel (VUB), Dienst ELEM, B-1050 Brussels, Belgium

K.B.M. Mahn

Department of Physics and Astronomy, Michigan State University, East Lansing, MI 48824, USA

S. Mancina

Department of Physics and Wisconsin IceCube Particle Astrophysics Center, University of Wisconsin, Madison, WI 53706, USA

M. Mandelartz

Fakultät für Physik \& Astronomie, Ruhr-Universität Bochum, D-44780 Bochum, Germany

R. Maruyama

Department of Physics, Yale University, New Haven, CT 06520, USA

K. Mase

Department of Physics and Institute for Global Prominent Research, Chiba University, Chiba 263-8522, Japan

R. Maunu 
Department of Physics, University of Maryland, College Park, MD 20742, USA

F. McNally

Department of Physics and Wisconsin IceCube Particle Astrophysics Center, University of Wisconsin, Madison, WI 53706, USA

K. Meagher

Université Libre de Bruxelles, Science Faculty CP230, B-1050 Brussels, Belgium

M. Medici

Niels Bohr Institute, University of Copenhagen, DK-2100 Copenhagen, Denmark

M. Meier

Department of Physics, TU Dortmund University, D-44221 Dortmund, Germany

A. Meli

Department of Physics and Astronomy, University of Gent, B-9000 Gent, Belgium

T. Menne

Department of Physics, TU Dortmund University, D-44221 Dortmund, Germany

G. Merino

Department of Physics and Wisconsin IceCube Particle Astrophysics Center, University of Wisconsin, Madison, WI 53706, USA

T. Meures

Université Libre de Bruxelles, Science Faculty CP230, B-1050 Brussels, Belgium

S. Miarecki

Lawrence Berkeley National Laboratory, Berkeley, CA 94720, USA

Deaprtment. of Physics, University of California, Berkeley, CA 94720, USA

T. Montaruli

Département de physique nucléaire et corpusculaire, Université de Genève, $\mathrm{CH}-1211$ Genève, Switzerland

M. Moulai

Department of Physics, Massachusetts Institute of Technology, Cambridge, MA 02139, USA

R. Nahnhauer

DESY, D-15735 Zeuthen, Germany

U. Naumann

Department of Physics, University of Wuppertal, D-42119 Wuppertal, Germany

G. Neer

Department of Physics and Astronomy, Michigan State University, East Lansing, MI 48824, USA

$\mathrm{H}$. Niederhausen

Department of Physics and Astronomy, Stony Brook University, Stony Brook, NY 11794-3800, USA

S.C. Nowicki

Department of Physics, University of Alberta, Edmonton, Alberta, T6G 2E1, Canada

D.R. Nygren

Lawrence Berkeley National Laboratory, Berkeley, CA 94720, USA

A. Obertacke Pollmann

Department of Physics, University of Wuppertal, D-42119 Wuppertal, Germany

A. Olivas

Department of Physics, University of Maryland, College Park, MD 20742, USA

A. O'Murchadha

Université Libre de Bruxelles, Science Faculty CP230, B-1050 Brussels, Belgium 


\section{T. Palczewski}

Lawrence Berkeley National Laboratory, Berkeley, CA 94720, USA

Department. of Physics, University of California, Berkeley, CA 94720, USA

H. Pandya

Bartol Research Institute and Department of Physics and Astronomy, University of Delaware, Newark, DE 19716, USA

D.V. Pankova

Department of Physics, Pennsylvania State University, University Park, PA 16802, USA

P. Peiffer

Institute of Physics, University of Mainz, Staudinger Weg 7, D-55099 Mainz, Germany

Ö. Penek

III. Physikalisches Institut, RWTH Aachen University, D-52056 Aachen, Germany

J.A. Pepper

Department of Physics and Astronomy, University of Alabama, Tuscaloosa, AL 35487, USA

C. Pérez de los Heros

Department of Physics and Astronomy, Uppsala University, Box 516, S-75120 Uppsala, Sweden

D. Pieloth

Department of Physics, TU Dortmund University, D-44221 Dortmund, Germany

E. Pinat

Université Libre de Bruxelles, Science Faculty CP230, B-1050 Brussels, Belgium

P.B. Price

Department of Physics, University of California, Berkeley, CA 94720, USA

G.T. Przybylski

Lawrence Berkeley National Laboratory, Berkeley, CA 94720, USA

M. Quinnan

Department of Physics, Pennsylvania State University, University Park, PA 16802, USA

C. Raab

Université Libre de Bruxelles, Science Faculty CP230, B-1050 Brussels, Belgium

L. Rädel

III. Physikalisches Institut, RWTH Aachen University, D-52056 Aachen, Germany

M. Rameez

Niels Bohr Institute, University of Copenhagen, DK-2100 Copenhagen, Denmark

K. Rawlins

Department of Physics and Astronomy, University of Alaska Anchorage, 3211 Providence Dr., Anchorage, AK 99508, USA

R. Reimann

III. Physikalisches Institut, RWTH Aachen University, D-52056 Aachen, Germany

B. Relethford

Department of Physics, Drexel University, 3141 Chestnut Street, Philadelphia, PA 19104, USA

M. Relich

Department of Physics and Institute for Global Prominent Research, Chiba University, Chiba 263-8522, Japan

E. Resconi

Physik-department, Technische Universität München, D-85748 Garching, Germany

W. Rhode 
Department of Physics, TU Dortmund University, D-44221 Dortmund, Germany

M. Richman

Department of Physics, Drexel University, 3141 Chestnut Street, Philadelphia, PA 19104, USA

B. Riedel

Department of Physics, University of Alberta, Edmonton, Alberta, T6G 2E1, Canada

S. Robertson

Department of Physics, University of Adelaide, Adelaide, 5005, Australia

M. Rongen

III. Physikalisches Institut, RWTH Aachen University, D-52056 Aachen, Germany

C. Rott

Department of Physics, Sungkyunkwan University, Suwon 440-746, Korea

T. Ruhe

Department of Physics, TU Dortmund University, D-44221 Dortmund, Germany

D. Ryckbosch

Department of Physics and Astronomy, University of Gent, B-9000 Gent, Belgium

D. Rysewyk

Department of Physics and Astronomy, Michigan State University, East Lansing, MI 48824, USA

L. Sabbatini

Department of Physics and Wisconsin IceCube Particle Astrophysics Center, University of Wisconsin, Madison, WI 53706, USA

S.E. Sanchez Herrera

Department of Physics, University of Alberta, Edmonton, Alberta, T6G 2E1, Canada

A. Sandrock

Department of Physics, TU Dortmund University, D-44221 Dortmund, Germany

J. Sandroos

Institute of Physics, University of Mainz, Staudinger Weg 7, D-55099 Mainz, Germany

S. Sarkar

Niels Bohr Institute, University of Copenhagen, DK-2100 Copenhagen, Denmark

Department of Physics, University of Oxford, 1 Keble Road, Oxford OX1 3NP, UK

K. Satalecka

DESY, D-15735 Zeuthen, Germany

P. Schlunder

Department of Physics, TU Dortmund University, D-44221 Dortmund, Germany

T. Schmidt

Department of Physics, University of Maryland, College Park, MD 20742, USA

S. Schoenen

III. Physikalisches Institut, RWTH Aachen University, D-52056 Aachen, Germany

S. Schöneberg

Fakultät für Physik \& Astronomie, Ruhr-Universität Bochum, D-44780 Bochum, Germany

L. Schumacher

III. Physikalisches Institut, RWTH Aachen University, D-52056 Aachen, Germany

D. Seckel

Bartol Research Institute and Department of Physics and Astronomy, University of Delaware, Newark, DE 19716, USA 


\section{S. Seunarine}

Department of Physics, University of Wisconsin, River Falls, WI 54022, USA

D. Soldin

Department of Physics, University of Wuppertal, D-42119 Wuppertal, Germany

M. Song

Department of Physics, University of Maryland, College Park, MD 20742, USA

G.M. Spiczak

Department of Physics, University of Wisconsin, River Falls, WI 54022, USA

\section{Spiering}

DESY, D-15735 Zeuthen, Germany

T. Stanev

Bartol Research Institute and Department of Physics and Astronomy, University of Delaware, Newark, DE 19716, USA

A. Stasik

DESY, D-15735 Zeuthen, Germany

\section{J. Stettner}

III. Physikalisches Institut, RWTH Aachen University, D-52056 Aachen, Germany

A. Steuer

Institute of Physics, University of Mainz, Staudinger Weg 7, D-55099 Mainz, Germany

T. Stezelberger

Lawrence Berkeley National Laboratory, Berkeley, CA 94720, USA

\section{R.G. Stokstad}

Lawrence Berkeley National Laboratory, Berkeley, CA 94720, USA

A. Stöß।

Department of Physics and Institute for Global Prominent Research, Chiba University, Chiba 263-8522, Japan

R. Ström

Department of Physics and Astronomy, Uppsala University, Box 516, S-75120 Uppsala, Sweden

N.L. Strotjohann

DESY, D-15735 Zeuthen, Germany

G.W. Sullivan

Department of Physics, University of Maryland, College Park, MD 20742, USA

M. Sutherland

Department of Physics and Center for Cosmology and Astro-Particle Physics, Ohio State University, Columbus, OH 43210, USA

H. Taavola

Department of Physics and Astronomy, Uppsala University, Box 516, S-75120 Uppsala, Sweden

\section{Taboada}

School of Physics and Center for Relativistic Astrophysics, Georgia Institute of Technology, Atlanta, GA 30332, USA

J. Tatar

Lawrence Berkeley National Laboratory, Berkeley, CA 94720, USA

Department of Physics, University of California, Berkeley, CA 94720, USA

F. Tenholt

Fakultät für Physik \& Astronomie, Ruhr-Universität Bochum, D-44780 Bochum, Germany

S. Ter-Antonyan 
Department of Physics, Southern University, Baton Rouge, LA 70813, USA

A. Terliuk

DESY, D-15735 Zeuthen, Germany

G. Tešić

Department of Physics, Pennsylvania State University, University Park, PA 16802, USA

S. Tilav

Bartol Research Institute and Department of Physics and Astronomy, University of Delaware, Newark, DE 19716, USA

P.A. Toale

Department of Physics and Astronomy, University of Alabama, Tuscaloosa, AL 35487, USA

M.N. Tobin

Department of Physics and Wisconsin IceCube Particle Astrophysics Center, University of Wisconsin, Madison, WI 53706, USA

S. Toscano

Vrije Universiteit Brussel (VUB), Dienst ELEM, B-1050 Brussels, Belgium

D. Tosi

Department of Physics and Wisconsin IceCube Particle Astrophysics Center, University of Wisconsin, Madison, WI 53706, USA

M. Tselengidou

Erlangen Centre for Astroparticle Physics, Friedrich-Alexander-Universität Erlangen-Nürnberg, D-91058 Erlangen, Germany

A. Turcati

Physik-department, Technische Universität München, D-85748 Garching, Germany

E. Unger

Department of Physics and Astronomy, Uppsala University, Box 516, S-75120 Uppsala, Sweden

M. Usner

DESY, D-15735 Zeuthen, Germany

J. Vandenbroucke

Department of Physics and Wisconsin IceCube Particle Astrophysics Center, University of Wisconsin, Madison, WI 53706, USA

N. van Eijndhoven

Vrije Universiteit Brussel (VUB), Dienst ELEM, B-1050 Brussels, Belgium

S. Vanheule

Department of Physics and Astronomy, University of Gent, B-9000 Gent, Belgium

M. van Rossem

Department of Physics and Wisconsin IceCube Particle Astrophysics Center, University of Wisconsin, Madison, WI 53706, USA

J. van Santen

DESY, D-15735 Zeuthen, Germany

M. Vehring

III. Physikalisches Institut, RWTH Aachen University, D-52056 Aachen, Germany

M. Voge

Physikalisches Institut, Universität Bonn, Nussallee 12, D-53115 Bonn, Germany

E. Vogel

III. Physikalisches Institut, RWTH Aachen University, D-52056 Aachen, Germany 
M. Vraeghe

Department of Physics and Astronomy, University of Gent, B-9000 Gent, Belgium

C. Walck

Oskar Klein Centre and Department of Physics, Stockholm University, SE-10691 Stockholm, Sweden

A. Wallace

Department of Physics, University of Adelaide, Adelaide, 5005, Australia

M. Wallraff

III. Physikalisches Institut, RWTH Aachen University, D-52056 Aachen, Germany

N. Wandkowsky

Department of Physics and Wisconsin IceCube Particle Astrophysics Center, University of Wisconsin, Madison, WI 53706, USA

Ch. Weaver

Department of Physics, University of Alberta, Edmonton, Alberta, T6G 2E1, Canada

M.J. Weiss

Department of Physics, Pennsylvania State University, University Park, PA 16802, USA

C. Wendt

Department of Physics and Wisconsin IceCube Particle Astrophysics Center, University of Wisconsin, Madison, WI 53706, USA

S. Westerhoff

Department of Physics and Wisconsin IceCube Particle Astrophysics Center, University of Wisconsin, Madison, WI 53706, USA

B.J. Whelan

Department of Physics, University of Adelaide, Adelaide, 5005, Australia

S. Wickmann

III. Physikalisches Institut, RWTH Aachen University, D-52056 Aachen, Germany

K. Wiebe

Institute of Physics, University of Mainz, Staudinger Weg 7, D-55099 Mainz, Germany

C.H. Wiebusch

III. Physikalisches Institut, RWTH Aachen University, D-52056 Aachen, Germany

L. Wille

Department of Physics and Wisconsin IceCube Particle Astrophysics Center, University of Wisconsin, Madison, WI 53706, USA

D.R. Williams

Department of Physics and Astronomy, University of Alabama, Tuscaloosa, AL 35487, USA

L. Wills

Department of Physics, Drexel University, 3141 Chestnut Street, Philadelphia, PA 19104, USA

M. Wolf

Oskar Klein Centre and Department of Physics, Stockholm University, SE-10691 Stockholm, Sweden

T.R. Wood

Department of Physics, University of Alberta, Edmonton, Alberta, T6G 2E1, Canada

E. Woolsey

Department of Physics, University of Alberta, Edmonton, Alberta, T6G 2E1, Canada

K. Woschnagg

Department of Physics, University of California, Berkeley, CA 94720, USA 
D.L. Xu

Department of Physics and Wisconsin IceCube Particle Astrophysics Center, University of Wisconsin, Madison, WI 53706, USA

X.W. Xu

Department of Physics, Southern University, Baton Rouge, LA 70813, USA

Y. Xu

Department of Physics and Astronomy, Stony Brook University, Stony Brook, NY 11794-3800, USA

J.P. Yanez

Department of Physics, University of Alberta, Edmonton, Alberta, T6G 2E1, Canada

G. Yodh

Department of Physics and Astronomy, University of California, Irvine, CA 92697, USA

S. Yoshida

Department of Physics and Institute for Global Prominent Research, Chiba University, Chiba 263-8522, Japan

M. Zoll

Oskar Klein Centre and Dept. of Physics, Stockholm University, SE-10691 Stockholm, Sweden

\section{Abstract}

Although high-energy astrophysical neutrinos were discovered in 2013, their origin is still unknown. Aiming for the identification of an electromagnetic counterpart of a rapidly fading source, we have implemented a realtime analysis framework for the IceCube neutrino observatory. Several analyses selecting neutrinos of astrophysical origin are now operating in realtime at the detector site in Antarctica and are producing alerts for the community to enable rapid follow-up observations. The goal of these observations is to locate the astrophysical objects responsible for these neutrino signals. This paper highlights the infrastructure in place both at the South Pole site and at IceCube facilities in the north that have enabled this fast follow-up program to be implemented. Additionally, this paper presents the first realtime analyses to be activated within this framework, highlights their sensitivities to astrophysical neutrinos and background event rates, and presents an outlook for future discoveries.

\section{Keywords}

Neutrino astronomy; Neutrino detectors; Transient sources; Multi-messenger astronomy

\section{Introduction}

Multimessenger astronomy, the combination of observations in cosmic rays, neutrinos, photons of all wavelengths, and gravitational waves, represents a powerful tool to study the physical processes driving the non-thermal universe. Neutrinos play an important role in this emerging field. The detection of a diffuse flux of astrophysical neutrinos by IceCube $\underline{1,2}$ with no clearly identified sources further motivates a multimessenger approach. Unlike their counterparts in photons and charged cosmic rays, neutrinos' low cross section and absence of electric charge allow them to travel the cosmological distances necessary to reach Earth from source regions without absorption or deflection. Observation of these astrophysical neutrinos can provide critical directional information that can be used to direct follow-up observations. Additionally, the detection of neutrinos from a source is a telltale sign of high-energy hadronic interactions. This feature could lead to the elucidation of the accelerating mechanism which produces 
the most energetic particles observed in the Universe, the highest energy cosmic rays. ${ }^{-}$Several models $\$, 5,6$ predict emission from flaring objects or other transient phenomena, requiring a rapid start for follow-up observations to be successful. This paper presents the IceCube realtime system, which enables rapid identification of neutrino candidates and issues notifications to follow-up observatories.

The IceCube neutrino detector,$\underline{7,8}$ consists of 86 strings, each instrumented with 60 digital optical modules (DOMs) spaced up to $17 \mathrm{~m}$ apart over a total vertical length of one kilometer. Strings are arranged in a hexagonal pattern with $125 \mathrm{~m}$ average horizontal spacing between neighboring strings. Eight of the deployed strings fill in a central volume between standard strings and create the more densely instrumented DeepCore region. - The deepest modules are located $2.45 \mathrm{~km}$ below the surface so that the instrument is shielded from the large background of cosmic rays at the surface by approximately $2 \mathrm{~km}$ of ice. Each DOM consists of a glass pressure housing containing the photomultiplier and electronics that independently digitize the signals using onboard electronics. The total instrumented detector volume is a cubic kilometer of highly transparent $\underline{\underline{10}}$ Antarctic ice.

IceCube does not directly observe neutrinos, but rather the Cherenkov emissions from secondary charged particles produced through electromagnetic interactions as these secondary particles travel through the Antarctic glacial ice. Therefore the ability to reconstruct accurately the direction of an event recorded in IceCube is highly dependent on the ability to reconstruct these secondary particles.

These secondary particles can produce two distinct classes of signals within IceCube. Track events are produced by muons, arising mainly from the charged current interaction of muon-type neutrinos, which produce

$(\mathrm{km})$ long light emission regions as they transit the detector. These tracks can be reconstructed with a directional uncertainty less than $1^{\circ}$, but with large energy uncertainty since an unknown fraction of their energy is deposited outside the instrumented volume. Shower events are produced by the charged current interaction of electron and tau-type neutrinos and by neutral current interactions of all neutrino types. Shower events tend to deposit all their energy within $(10 \mathrm{~m})$, producing a relatively isotropic deposition of light emission. These types of events tend to have good energy resolution $\left(\delta E / E \sim 15 \%{ }_{11}\right)$, but have limited angular reconstruction in ice, with typical angular resolutions on the order of $10-$ $15^{\circ} \cdot \underline{12}$

The depth of the detector and its size result in a trigger rate of approximately $2.7 \mathrm{kHz}$ for penetrating muons produced by interactions of cosmic rays in the atmosphere above the detector. The neutrino detection rate (a few $\mathrm{mHz}$ ) is dominated by neutrinos produced in the Earth's atmosphere. This large down-going background in the southern hemisphere necessitates a higher threshold for neutrino detection relative to the earth-shielded northern hemisphere. The first challenge of the realtime alert system is to select a sufficiently pure sample of neutrinos, while the second is to identify the small fraction of neutrinos that are likely to be astrophysical in origin.

IceCube has sensitivity to astrophysical neutrinos from the entire sky (4 $\pi$ steradians) and operates with a high duty factor (> 99\%), putting it in a unique position to act as a trigger for other observatories around the globe. Given the limited field of view of most follow-up instruments, events from the track event class are preferred for realtime follow-up alerts. The smaller directional uncertainties of track events also help to limit coincidental discoveries when sensitive telescopes are pointed at unexplored regions of the sky. 
IceCube has long had an active follow-up program. For several years, alerts have been sent out to optical, gamma-ray, and X-ray telescope, $\frac{13}{\underline{13}}$ which has led to a number of interesting results $\underline{\underline{14}, 15,16,17,18}$ as well as fostering a rich collaboration between electromagnetic and neutrino observatories. These longrunning follow-up programs are supplemented by new neutrino selections that target single events deemed likely to be of astrophysical origin in the IceCube realtime alert system.

This paper describes the technical infrastructure (Section 2) now in place at the South Pole and at IceCube's computing facilities in the northern hemisphere, as well as the practical challenges of working with a remote detector to support the realtime alert system. It highlights the existing follow-up programs that are now in operation (Section 3 ) in this realtime alert framework and issuing alerts for astrophysical neutrino candidates to follow-up observatories. These alerts, such as, $\underline{\underline{19}}$ have received prompt observations by many observatories across the electromagnetic spectrum.

\section{Follow-up infrastucture}

\subsection{Processing at the South Pole}

Due to the remote Antarctic location, IceCube has established a set of automated data collection and filtering systems that process all data received from its DOMs. These systems are responsible for collecting correlated data records from DOMs, known as an event, application of calibration information, processing waveform data from the DOMs to reconstruct tracks and shower events, and application of event selections to select events for consideration in neutrino search algorithms. These systems run continuously, processing data from the DOMs as rapidly as possible on a dedicated computing cluster located at the detector site. These systems also host event selections used to identify astrophysical neutrino events, generate alert messages, monitor the health of the IceCube detector, and transmit this information north for dissemination to the astronomical community with minimal delay.

The IceCube data acquisition (DAQ) system is responsible for managing communication with and control of all deployed DOMs. $\underline{20}, \underline{1}$ Time-stamped signals from all DOMs are received by the DAQ. $\underline{\underline{8}}$ The primary trigger for neutrino alerts searches for $8 \mathrm{DOMs}$ receiving photon signals in a $5 \mu \mathrm{s}$ time window. Once the trigger has been satisfied, the DAQ records all DOM signals from $a+6 /$

$4 \mu s$ window around the trigger time into a single event. The recorded events from each $\sim 1 \mathrm{~s}$ slice of data are immediately made available to the online processing and filtering system.

The online processing and filtering system ${ }^{8}$ distributes each event to one member of a farm of $\sim 400$ identical, dedicated calibration and filtering client processors. These clients include software for calibration of DOM digitized waveforms and extraction of light arrival times from the recorded DOM waveforms. The photon arrival and amplitude information is extracted from the calibrated waveforms using the DOM response to single photons using a non-negative linear least squares algorithm. $\frac{11}{}$ The relative timing of the DOM signals is calibrated by the DAQ to UTC times with a measured accuracy of $1.2 \mathrm{~ns} \cdot \frac{-8}{-}$ The calibration values used online are identical to those used in offline analyses, and show little year-to-year variation.

This system also includes several reconstruction algorithms that characterize each event's extracted light arrival information against the expected patterns from track and shower events to determine the direction, position and energy of each event. $\stackrel{11}{ }$ Based on these reconstructions, approximately $1 \%$ of these events are selected to be potentially of neutrino origin and are processed with additional, more 
sophisticated and computationally intensive reconstructions (the "OnlineL2" selection). The selection targets well-reconstructed, track-like events with a charge threshold that depends on the reconstructed track direction, with more stringent cuts applied in the atmospheric muon dominated down-going region. $\frac{17}{}$ The OnlineL2 selection has been in operation since 2011 with each year seeing incremental improvements that bring better tools developed offline to the online system. For the gamma-ray, optical, and x-ray follow-up program (see Section 3.1) the OnlineL2 selection serves as the pre-selection for these online neutrino searches.

The online processing and filtering clients are able to select any triggered events that pass established event quality, energy, and topology criteria for the alert systems based on the online event reconstruction information. These selection criteria search for single events, such as the rare highenergy astrophysical neutrinos, are established and verified in offline studies, and are derived from similar selections used in published analyses. $.2,23, \underline{24,} \underline{25}$ These selections include a high-energy starting event selection (see Section 3.2) and an extremely high-energy track selection (see Section 3.3).

The results from this client farm are returned to the online processing and filtering system, where data files containing all events are created and archived. Each event is processed in the order received, maintaining a strict first-in, first-out order. This architecture puts practical limits on the complexity of reconstructions performed in the filtering client as all events must be reconstructed within $\sim 30 \mathrm{~s}$ to prevent pileup. Several computationally complex reconstructions performed offline $\frac{11}{1}$ can require minutes to hours to evaluate a single event and are not supportable by the limited computational power available at the South Pole. Events passing the online alert system criteria are forwarded to a dedicated online alert system. Typically, alert information is presented to the online alert system with a delay of about $\sim 20 \mathrm{~s}$.

The online alert system receives events selected for alert generation and immediately creates messages for transmission to the northern hemisphere data center. The first message contains a short JSONformatted ${ }^{1}$ message containing the critical information needed for inclusion in automatic alerts to astronomical partners (event time, run information, direction and energy). A second, larger message is generated encoding a compact data record of the extracted light arrival information from the DOMs. This second data record is also transmitted north in order to start more computationally intensive follow-up reconstructions and alert quality verification checks. This splitting of alert information across multiple messages is done to avoid transmission delays in the critical information that is used in the initial automatic alerts.

To ensure IceCube is operating in a stable manner before notifying follow-up instruments, the DAQ and the online processing and filtering system track several detector health monitoring quantities. These include the rate of triggered events, rate of filtered events, number of active DOMs, and several other detector environmental criteria that have been found to be good indicators of detector health and data quality.

\subsection{Data transfer}

Alert messages from the online alert system and all detector health information is reported to the detector experiment control system, IceCube Live (I3Live). ${ }^{-}$The I3Live system has several data transport methods available to move information from Antarctica to the primary data center at the University of Wisconsin, Madison, each with different bandwidth, message size limits, and latency. 
JSON messages containing the alert summary information, as well as messages containing compact records of light arrival information, are transferred by the Iridium RUDICS system. $\stackrel{2}{-}$ The IceCube detector utilizes 2 links, each with $2.4 \mathrm{kbps}$ bandwidth. Messages are sent north without message size restrictions, but can experience some delays due to higher priority data traffic. Total message latencies, including the $\sim 20 \mathrm{~s}$ event processing and filtering time, are shown in Fig. 1 for the short JSON alert messages, with a median total message latency of $33 \mathrm{~s}$.

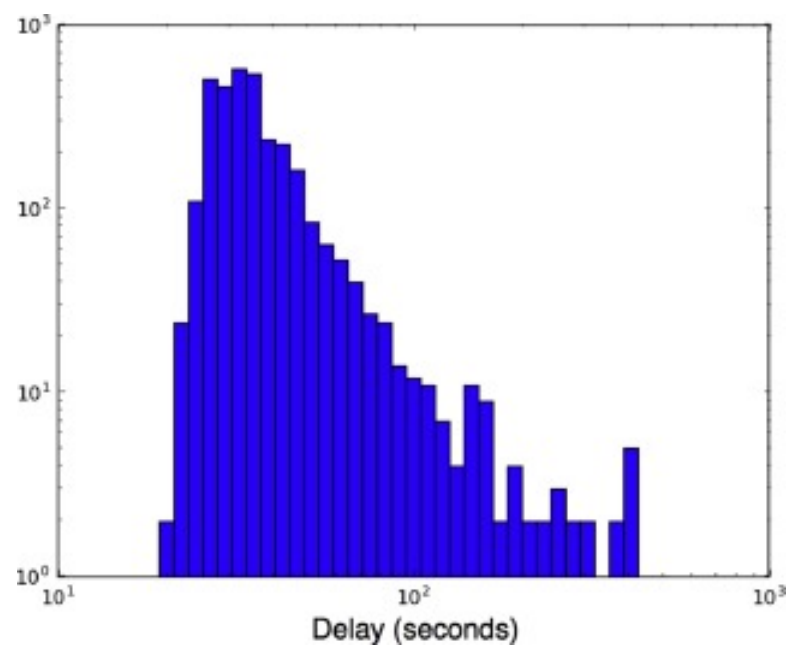

Fig. 1. Histogram of total alert message latency for the Iridium RUDICS data messaging system, measured from time the event triggers the data acquisition system at the South Pole until the alert is received in the northern hemisphere data center. The median message latency is $33 \mathrm{~s}$.

\subsection{Alert generation}

Alert messages arriving from South Pole via I3Live are immediately stored in a dedicated database and distributed to a set of follow-up analysis clients. This distribution employs a $\mathrm{ZMQ}^{\underline{3}}$ framework with a publisher-subscriber model, where the I3Live acts as a publisher, distributing the alert information to all analysis clients which have subscribed to a particular type of alert message. This setup provides a scalable and stable platform for distributing event streams of varying rates to a multitude of clients, while decoupling the operation of subscribers from each other and the publisher, allowing each subscriber to operate in an independent manner.

The follow-up clients use a shared library, which provides methods to communicate with the event publisher, assess the detector status, do basic analysis tasks and generate properly formatted alert messages to other observatories using several forms of automated communication, such as email or VOEvent $\frac{26}{}$ messages.

Each realtime analysis has a dedicated follow-up client process that is triggered by the arrival of an incoming event and determines whether an alert should be generated. These follow-up clients have the ability to fetch previously recorded events from the database for correlation studies, as well as to monitor quantities vital to the operation of the detector to ensure that certain data quality criteria are met. Depending on the observation plan established with the follow-up observatory, the alert generation can be inhibited, if the source is not visible to the observatory at the time of the alert or during the following nights. 


\subsubsection{Detector stability monitoring}

All event selections and alert mechanisms depend on events being acquired in well-determined and well-understood operational states of the detector. For traditional offline analyses, periods of data taking are split in segments with a duration of eight hours, then each segment is manually inspected to ensure good data quality. Data quality can be impacted by several operational issues, such as groups of DOMs or entire strings failing to deliver data, or by operation of light-generating calibration devices within the detector.

To ensure a quick alert generation, realtime alerts cannot rely on manual determination of data quality. Therefore an automated system to monitor the detector stability continuously has been implemented. $\underline{\underline{17}}$ There are three ingredients, which are directly related to the different stages of the event selection (see Section 2.1):

- The rate of primary DAQ triggers.

- The rate of the well-defined muon track events selected by the online filter system.

- The rate of the events selected by the OnlineL2 selection.

These quantities are recorded in ten minute intervals. An exponentially weighted moving average is formed over past measurements and the rates in each new bin are compared to this average (weighted with their statistical uncertainty). The deviation from the long term average yields a stability score from which the goodness of the data can be measured. An example of one day of mostly stable conditions with two outages in between is given in Fig. 2.

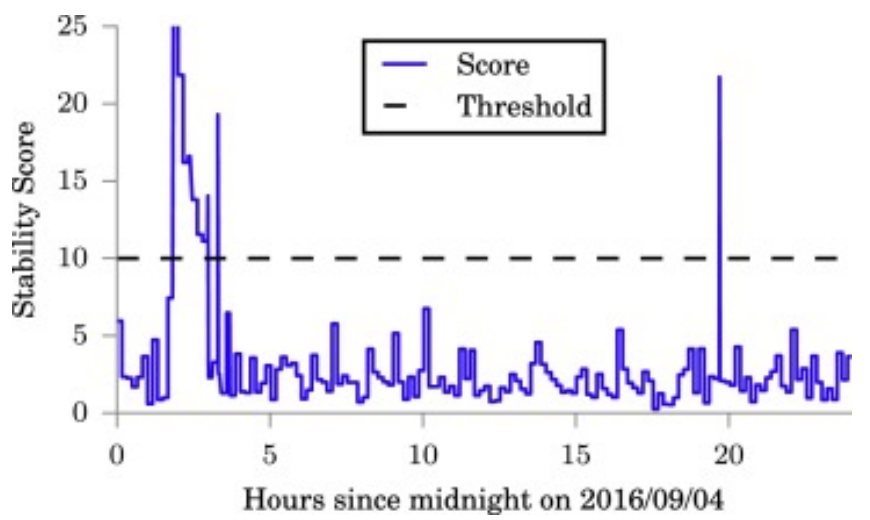

Fig. 2. Output of the online stability monitoring from an anomalous day. The stability score encodes information about the quality of the online data taking. In this example a faulty power supply (at hour $\sim 2$ ) and a full restart of the detector (at hour $\sim 19$ ) cause some downtime, where the stability score exceeds the threshold of 10, during otherwise stable operations. The bad intervals are correctly identified.

The difference in good quality data taking periods measured by the online criteria and the offline monitoring system is less than one percent, with the online monitoring rejecting slightly more time periods out of an abundance of caution.

\subsubsection{Alerts, revisions and retractions}

Alerts are issued to the observational community in several ways, both through public and private channels. Public alerts utilize the Astrophysical Multimessenger Observatory Network (AMON) system 27 
as a gateway to the Gamma-ray Coordinates Network (GCN) $\underline{28}$ and are immediately available to followup observatories. Each alert has a well-defined structure and content, which is published online. ${ }^{4}$ Private alert communication is generally done via electronic mail messages directly to observatory operation centers or by private distribution lists via AMON and GCN.

The event reconstruction information can be refined within a few hours of an initial alert with better angular reconstruction and improved distinction between track-like and shower-like events. In the initial alert, directional reconstructions from the OnlineL2 selection performed at the South Pole are used. Once the full event information arrives in the northern data center, additional reconstructions can begin on larger computer clusters. These CPU-intensive reconstructions evaluate the likelihood of different arrival directions using a series of ever-finer directional grids on the sky to determine the best-fit direction. Angular resolution estimates at different confidence levels are estimated using Wilks's theorem that has been calibrated using known angular errors measured with simulated neutrino data samples.

These refined reconstructions improve angular resolution by more than $50 \%$ (to $\leq 1^{\circ}$ for most tracks) and also provide information on the energy loss within the detector. The energy loss profile, in conjunction with the likelihood direction scans, provide important inputs to further distinguish between track-like and shower-like events. The angular resolution for shower-like events, typically $\sim 10-15^{\circ}$, is too large for follow-up with most telescopes.

Once these refined reconstructions are complete, a revision to the original alert can be created reflecting the updated event information. The revised alert retains the same event number information as the original alert, but the revision number is increased by 1 . Additionally, any alert that is determined to originate from misreconstructed background events or other instrumental effects will be retracted within hours following the original alert. Refined reconstructions for all high-energy starting events (see Section 3.2) and extremely high-energy track events (see Section 3.3) are automatically performed and are accompanied by alert revisions upon completion. Refined reconstructions are available for other events on an as-needed basis.

\section{Realtime alert systems}

IceCube presently operates several alert systems utilizing the realtime framework described in the preceding section. The X-ray, optical, and gamma-ray follow-up program described in Section 3.1 has been in operation for several years and is accompanied by two new online streams beginning in 2016. The first new alert system selects track-like high-energy starting events (HESE, Section 3.2) and the second alert system targets extremely high-energy through-going tracks (EHE, Section 3.3).

The infrastructure in place allows for independent, simultaneous operation of alert systems that search for different signals. The HESE and EHE alert systems both trigger on the detection of single events, while other follow-up programs are triggered by the accumulation of neutrino candidates consistent with coming from a single point in the sky. As new analysis techniques are developed to quickly isolate astrophysical neutrino candidates, they will be moved to the realtime alert system to generate triggers for interested follow-up observatories. 


\subsection{Gamma-ray, optical and X-ray follow-up}

The gamma-ray, optical and X-ray follow-up program is designed to detect bursts of several neutrino-like events that, when considered alone, would not be distinguishable from background. The main background for up-going events, i.e., events coming from the northern hemisphere, are atmospheric neutrinos. These neutrinos arise from decays of charged pions and kaons that are created by cosmic rays striking the atmosphere. For down-going events the background is dominated by lower energy muons from these atmospheric showers, so only high-energy tracks are selected. $\underline{22}$ These backgrounds are well understood, with well measured rates and isotropic angular distributions in any selected zenith range. This follow-up program searches for statistically significant clustering in time and space of the observed neutrino candidates, and uses any spatial and time correlation as an indication of a potential neutrino burst. Given the low expected rate of alerts from true neutrino bursts, alert thresholds are set to generate follow-up alerts for a few background over-fluctuations per year. A common event selection, described in Section 3.1.1 is used in two different neutrino burst time scale searches, optical and X-ray follow-up (OFU, Section 3.1.2), searching time scales up to $100 \mathrm{~s}$, and the gamma-ray follow-up (GFU, Section 3.1.3), searching time scales up to 3 weeks.

\subsubsection{Event selection}

Both OFU and GFU are based on the same neutrino event selection. This selection starts with the OnlineL2 pre-selection (Section 2.1) which selects tracks that are potentially neutrino generated and contains results from more sophisticated track and energy reconstructions as well as enhanced angular uncertainty estimators. .22 Prior to May 2016, the OFU and GFU selections used independent event selections that yielded similar event samples, but they have since been unified into the single selection described here. The results of these reconstructions are used as the input to a multivariate classifier. Further reduction of the atmospheric muon background and separation of an astrophysical signal is

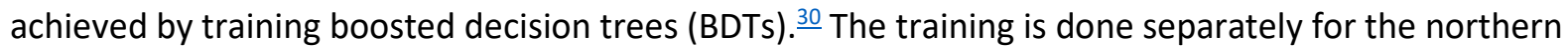
and southern hemisphere to account for the different kinds of background encountered in each region, yielding a final event selection rate of $5 \mathrm{mHz}$, equally divided between both hemispheres. A description of the variables entering the BDTs can be found in..$^{17}$

Two different subsamples are defined from the output of the BDTs:

- The OFU subsample, limited to up-going tracks from the northern sky, uses a relaxed BDT cut, with an event rate in this hemisphere that is slightly higher $(3 \mathrm{mHz})$ than for the GFU analysis.

- The GFU subsample selects tracks from the entire sky. In the northern sky, the BDT cut is more selective than that of the OFU analysis, leading to a lower event rate $(2 \mathrm{mHz})$ in this region. The BDT cut in the southern sky is chosen such that the event rate is constant in all declinations and is matched to the northern sky.

The different BDT cuts are selected to achieve the best sensitivity to neutrinos for the different time scales searched relative to the expected backgrounds. A smaller search time window is used in the OFU analysis to reduce background events and to allow for the more inclusive event selection to be used. The effective areas for neutrinos, as used in the OFU and GFU selections, are shown in Fig. 3 . The angular error for selected neutrino events, and its dependence on the energy of the neutrino, is shown in Fig. 4. All events selected by the BDT for either OFU or GFU are immediately transferred from the South Pole to the north, where they are made available to the OFU and GFU analysis clients as described below. 

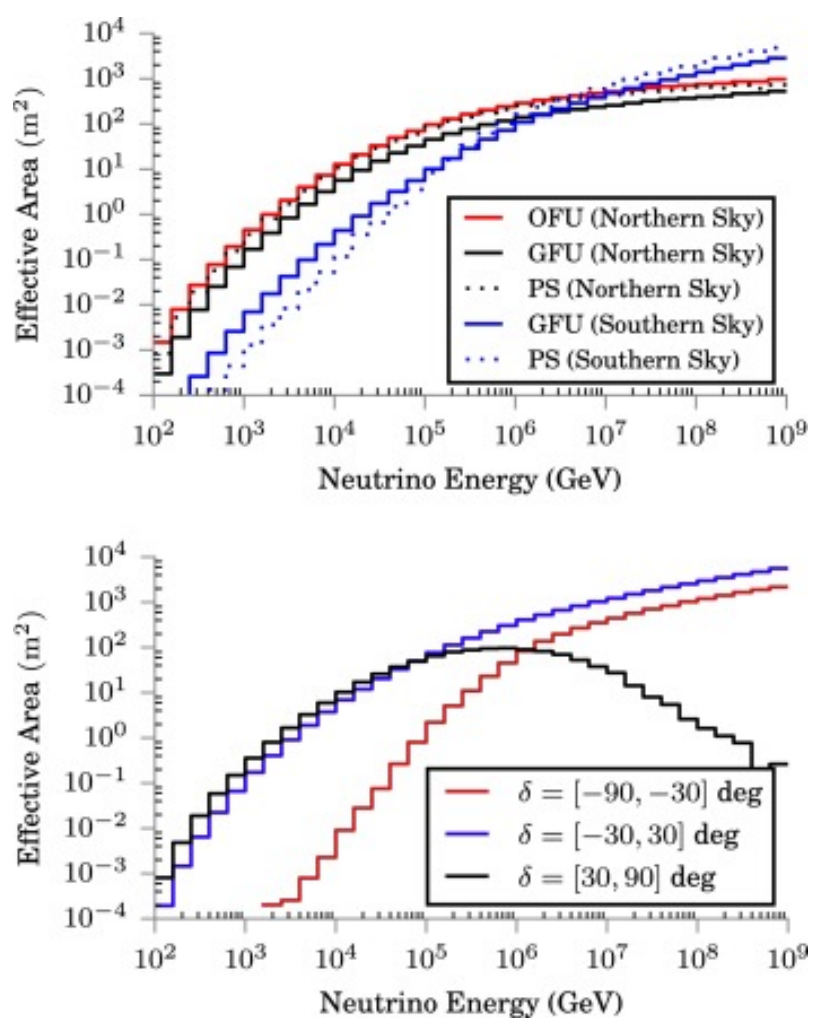

Fig. 3. Effective areas for different subsets of the online neutrino event selection. The upper panel compares the OFU and GFU subsamples to each other, as well as to the published point source search selections [29], and the lower panel compares different declination bands within the GFU subsample. The OFU subsample is only available in the northern sky, whereas the GFU subsample covers both hemispheres.
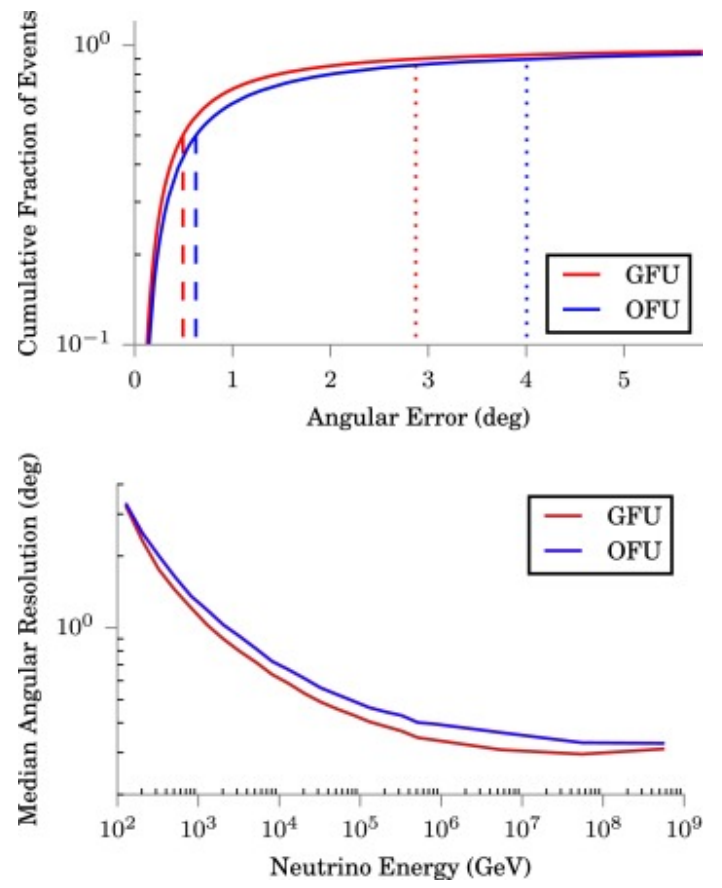

Fig. 4. Angular error of events in the GFU and OFU analyses. The angular error is the opening angle between the online reconstructed direction and the true direction of simulated neutrinos. The upper plot shows the 
cumulative distribution, with the dashed (dotted) line highlighting the 50\% (90\%) containment. The lower plot shows the median angular error as a function of the true neutrino energy.

\subsubsection{OFU alerts}

The optical and X-ray follow-up $\underline{14}, \underline{15}, \underline{16}$ aims for the real-time detection of neutrino bursts on time scales of up to $100 \mathrm{~s}$, which are predicted to be produced in gamma-ray bursts or supernovae with choked jets. $, 31,32$ Given the reduced background rate from the Earth's shielding of cosmic rays, the OFU program focuses on alerts from the northern hemisphere (see Fig. 3.) The program has been operating since December 2008 when interesting neutrino events were initially forwarded to the now-decommissioned ROTSE telescopes. $\frac{33}{-}$ Starting in August 2010, the Palomar Transient Factory $\underline{34}$ started receiving OFU alerts, and in February 2011 the inclusion of the Swift-XRT. $\frac{35}{5}$ targeting GRB afterglows, marked the expansion of the program to the X-ray regime. In 2016, the program was again expanded in its optical capabilities, enabling more complete sky coverage by distributing alerts also to the MASTER telescopes. $\frac{36}{}$ An extension to ASAS-SN $\frac{37}{}$ and $\mathrm{LCO}^{\underline{38}}$ telescopes is being planned.

OFU selects neutrino multiplets, requiring at least two events within $100 \mathrm{~s}$ and with an angular difference of less than $3.5^{\circ}$. Multiplets with a multiplicity larger than two are immediately forwarded to optical and X-ray telescopes. For doublets an additional quality cut is applied. This quality cut parameter, $\lambda$, is defined as follows:

$$
\lambda=\frac{\Delta \psi^{2}}{\sigma_{q}^{2}}+2 \ln \left(2 \pi \sigma_{q}^{2}\right)-2 \ln \left(1-\exp \left(-\frac{\theta_{A}^{2}}{2 \sigma_{w}^{2}}\right)\right)+2 \ln \left(\frac{\Delta t}{100 s}\right)
$$

where the time between the neutrinos in the doublet is denoted as $\Delta T$, and their angular separation as $\Delta \psi$. The quantities $\sigma_{q}^{2}=\sigma_{1}^{2}+\sigma_{2}^{2}$ and $\sigma_{w}^{2}=\left(1 / \sigma_{1}^{2}+1 / \sigma_{2}^{2}\right)^{-1}$ depend on the per-event estimated directional uncertainties $\sigma_{1}$ and $\sigma_{2}$ of the two neutrino events, typically $\sim 1^{\circ}$. The angle $\vartheta_{A}$ corresponds to the circularized angular radius of the field of view (FoV) of the follow-up telescope. The quality parameter $(\lambda)$ is smaller for more signal-like alerts, which have small separation $\Delta \psi$, small time difference $\Delta T$ and a high chance to lie in the FoV of the telescope. Thus, $\lambda$ is a useful parameter to identify signal-like doublets, and reduce the rate of background alerts. For each follow-up instrument, a specific cut on $\lambda$ is applied in order not to exceed the granted number of alerts per telescope and to send the most significant alerts to the follow-up instruments, as well as ensuring that any potential alert position is available for observation (e.g., at a sufficient distance from the Sun and Moon). The circularized angular radius $\left(\vartheta_{A}\right)$ and maximum allowed alerts per year determine the $\lambda$ cut level for each follow-up telescope. PTF and Master $\theta_{A}=0.9$ receive up to 7 alerts per year, while the Swift-XRT $\Theta_{A}=$ 0.5 receives up to 3 . Given the small angular aperture, Swift-XRT observations are done by tiled observations about the alert position. Longer term observations are scheduled as required based on the results of the initial observation.

The combined direction of the events in the multiplet and multiplet detection date and time are sent to the telescopes as an alert. The combined direction is the weighted arithmetic mean, weighting the individual directions with their inverse squared error, given by the per-event directional uncertainty $\sigma_{i}$. The error of the combined direction is given by $\Theta_{w}=\left(\sum_{i=1}^{N}\left(\frac{1}{\sigma_{i}}\right)^{2}\right)^{-1}$, where $N$ is the multiplicity of the alert. 


\subsubsection{GFU alerts}

The gamma-ray follow-up (GFU) searches for neutrino bursts on time scales of up to three weeks, tailored to the variability observed in several sources by imaging atmospheric Cherenkov telescopes. The GFU program is described in detail elsewhere. ${ }^{17}$ The GFU analysis searches for an excess of neutrino events in the vicinity of sources from a predefined source catalog. The list of monitored sources is based on the second Fermi point-source catalog, ${ }^{39}$ containing mostly BL-Lac objects and FSRQs, which have exhibited previous variable behavior and are visible to the follow-up telescopes. When a significant cluster is observed, the information is forwarded to the MAGIC $\underline{40}$ and VERITAS telescopes $\underline{41}$ (depending on source visibility at each site and distance from the Moon) to search for a coincident flare in very highenergy gamma rays. The program has been operating since 2012 with an online event selection covering the northern hemisphere sky. In 2015, it was extended to include the southern hemisphere sky.

A maximum-likelihood based search for point sources $\underline{42}$ is paired with a time-clustering search algorithm ${ }^{43}$ to search for neutrino bursts from a given source direction. Starting with the last event observed, time windows of up to three weeks in the past are tested to determine the most likely time frame of a flare, identified as a significant deviation of the number of detected neutrinos from the expected background. For each time window, the detector uptime (see Section 2.3.1) is considered in the likelihood calculation. A detailed description of the algorithm can be found in. 17

In early operation, the analysis used a binned counting method and a basic event selection which required event quality parameters to exceed a fixed threshold. With the aforementioned upgrade to BDTs and the maximum-likelihood search, the sensitivity has improved by $25 \%$ at the horizon and $65 \%$ towards the North Pole, which supports an increase in the number of monitored sources from 109 to 184 , by requiring a higher significance threshold for the alert generation. On average, two alerts per year are expected from background.

With the inclusion of the southern sky in the event selection, a collaboration with the HESS telescope ${ }^{44}$ is in preparation. Furthermore, future work will extend this program to flares arising from the entire sky on arbitrary time scales (the upper time scale is given by the results from the static point source search $^{22}$ ).

\subsection{HESE alerts}

The IceCube high-energy starting event (HESE) search has resulted in a clear detection $(>6.5 \sigma)$ of astrophysical neutrinos. $\underline{23,25, \underline{45}}$ However, the nature of the sources responsible for these neutrinos is not yet known. The sources of these neutrinos may be identified by the detection of an electromagnetic counterpart in rapid follow-up observations.

IceCube has detected 54 HESE neutrino candidates in 4 years of data. .5 These events have interaction vertices inside the detector fiducial volume and are classified in two main categories: track-like events from charged-current interactions of muon neutrinos (and potentially from the $\sim 18 \%$ of tau neutrino interactions that produce a high-energy muon) and shower-like events from all other interactions (neutral-current interactions and charged-current interactions of electron neutrinos and most tau neutrinos). The HESE data is dominated by shower-like events. In the 4-year data sample, there are 14 track-like events, while the remaining 40 are shower-like events. Given the better angular resolution of the track events, only the track-like events identified online are considered for HESE alerts and distributed publicly via AMON and the GCN network. 


\subsubsection{HESE track selection and alerts}

The astrophysical signal is most prominent at high energies where contained neutrino interactions result in a significant amount of light in the detector, and therefore a large amount of total charge detected by the DOMs. We use the total charge observed within $5 \mu \mathrm{s}$ of the event start time in order to cut out light from combined low energy events. All hits in the more densely instrumented DeepCore portion of the detector ${ }^{9}$ and on any single DOMs containing more than $50 \%$ of the total charge in the event are excluded to prevent the signal from a single DOM very close to a particle track from dominating the charge measurement. Here, only events with $\geq 6000$ photoelectrons are considered for HESE alerts. The primary background for HESE tracks arises from rare atmospheric muon events that evade the veto criteria. $\underline{25}$

Additionally, for an event to be considered as a HESE track, it must also exhibit signal-like and track-like characteristics. This is parameterized by the "signal_trackness" parameter, a number between 0 and 1. To calculate this number, Monte Carlo simulations including both signal and background events have been considered. Signal events were simulated with an energy spectrum of $E^{-2.58}$, the best fit spectrum observed by the IceCube HESE analysis. $\frac{45}{}$ A Bayesian approach has been used to calculate the probability that a HESE event is a track-like signal event:

Signal_Trackness $=\frac{f_{\text {track }} P_{\text {track }}}{f_{\text {track }} P_{\text {track }}+f_{\text {shower }} P_{\text {shower }}+\left(\frac{f_{\text {bkg }}}{f_{\text {sig }}}\right) P_{\text {bkg }}}$, where $P_{\text {track, }}, P_{\text {shower, }}$ and $P_{\text {bkg }}$ are the PDFs of log-likelihood ratios (value from the shower reconstruction divided by that of the track reconstruction) for track-like events, shower-like events, and backgrounds, respectively. The variables $f_{\text {track, }} f_{\text {shower, }} f_{\text {bkg, }}$ and $f_{\text {sig }}$ are the prior probabilities, with $f_{\text {track }}=1-f_{\text {shower }}$ and is given by:

$f_{\text {track }}=\frac{R_{\mu} R_{\mu, c c}+R_{r} R_{r, c c} R_{r, c c, \mu}}{R_{e}+R_{\mu}+R_{r}}$.

Based on studies of simulated events, $R_{e}: R_{\mu}: R_{\tau}$ is 2.48: 1.0: 1.52. Also, $R_{\mu, c c}=0.78, R_{r, c c}=0.86$ are the fractions of $v_{\mu}$ and $v_{\tau}$ events interacting via CC, respectively, and $R_{\tau, c c, \mu}$ is related to the branching ratio of $\tau \rightarrow \mu$. Note that this number is approximately half of the $\tau \rightarrow \mu$ branching ratio of 0.18 , because only half of the $\tau \rightarrow \mu$ decays put enough energy into the muon for it to be detected as a high energy muon. The quantity $f_{\mathrm{bkg}} / f_{\mathrm{sig}}$ is the ratio of the background to signal event rate that is dependent on charge.

Alerts are sent only for events having signal_trackness $\geq 0.1$. This yields about 1.1 signal-like track-like events from an $\mathrm{E}^{-2.58}$ astrophysical spectrum ${ }^{45}$ and about 3.7 total background events per year. Thirteen of the 14 manually identified track events in the published HESE samples $\underline{45}$ would also be identified as tracks by this signal_trackness criteria. The effective areas for this selection, for the entire sky and the northern and southern skies separately, are shown in Fig. 7.

Events passing these criteria generate a public alert via the GCN network sent via AMON. These alerts contain the best-fit source direction and uncertainty from the online reconstruction, date and time of the event, total measured charge, and signal_trackness value.

Studies of simulated HESE track events provide estimations of HESE angular errors in real-time. The angular separation between the true neutrino direction and online reconstructed direction is an estimate of how well our online reconstruction performs. Events with signal_trackness $\geq 0.1$ have a median angular error of $0.4^{\circ}-1.6^{\circ}\left(1.2^{\circ}-8.9^{\circ}\right.$ for $90 \%$ containment $)$ based on the properties of the individual events in the simulated HESE track samples. Events with larger track length inside the 
detector are better reconstructed and therefore have smaller angular errors. Events passing a minimum reconstructed track length in the detector of $200 \mathrm{~m}$ ( $80 \%$ of the events) have a median angular error of $0.55^{\circ}$ ( $1.89^{\circ}$ for $90 \%$ containment) as illustrated in Fig. 9 . Those events that do not pass a minimum track length cut (20\%), but still pass the signal_trackness selection, are reported with an upper limit fixed median angular error of $1.6^{\circ}$ ( $8.9^{\circ}$ for $90 \%$ containment). After detection and initial alert generation, follow-up reconstructions of the HESE events by computer clusters in the northern hemisphere are immediately started, and revised directional coordinates and improved angular uncertainty are released within a few hours of the initial alert (see Section 2.3.2).

\subsubsection{Options for increasing the signal-to-noise ratio}

Each HESE alert contains quantities that can be used by rapid follow-up observatories to increase the signal-to-noise ratio. Requiring a larger signal_trackness results in less background with some loss in signal efficiency. Fig. 5 shows the rate versus different cuts on signal_trackness for three different neutrino fluxes using simulated data: astrophysical track events in red, track-like atmospheric conventional neutrino background in green, and track-like atmospheric muon background in blue. Contributions from a prompt neutrino flux are expected to be small. $\underline{\underline{25}} \underline{\mathrm{Fig} .} \mathbf{5}$ also shows the rate (number of events per year) vs. different cuts on signal_trackness for signal and background simulated HESE events with charge $>7000$ p.e.
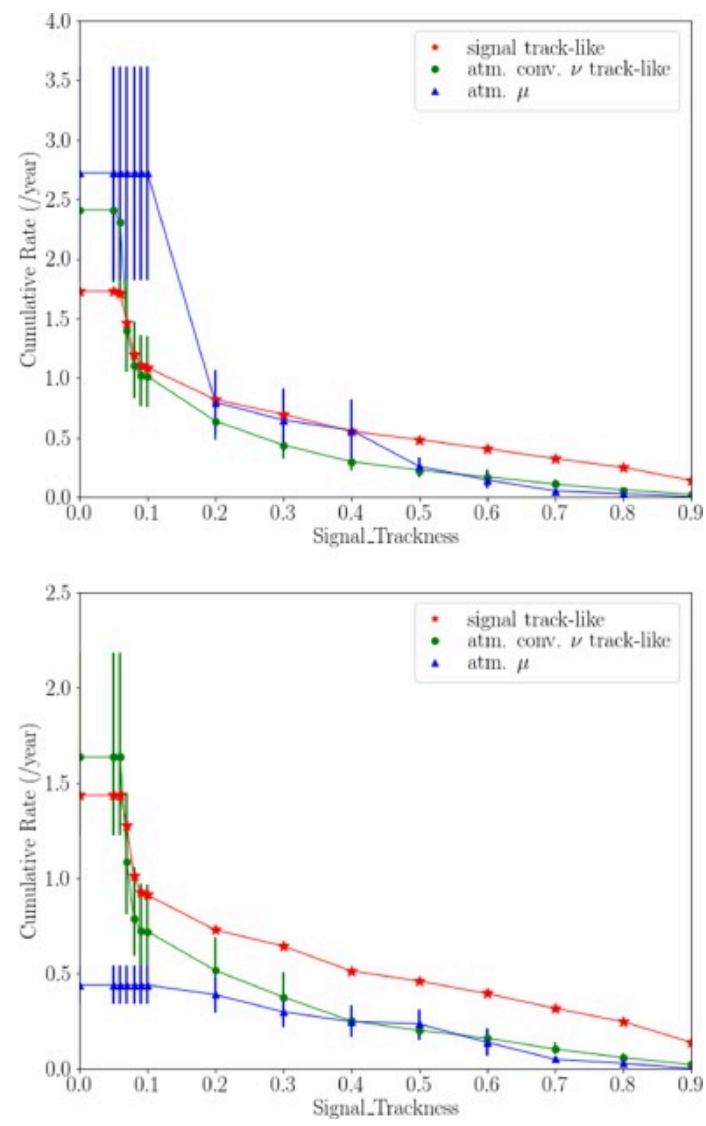

Fig. 5. Rate (number of events per year) vs. different cuts on the signal_trackness estimator for HESE signal and background with charge > 6000 p.e. (upper panel) and > 7000 p.e. (lower panel). HESE track events are shown in red, the atmospheric conventional neutrinos in green, and the atmospheric muons in blue. Error bars 
represent the standard deviation of the rates. The muon background error bars are larger than the other two components due to low statistics of the computationally-intensive simulation of the muon background.

Another option is to require larger deposited charge. Table 1 shows the signal and background rates (expected number of events per year) for different charge cuts as well as the signal to noise ratio (SNR). All numbers in this table are for events with signal_trackness $\geq 0.1$. The follow-up observatories can decide which events to observe based on their charge, signal_trackness, and rate information.

Table 1. Different cuts on HESE event charges, measured in photoelectrons, result in different signal, noise rates, and signal to noise ratios. In parentheses, the contribution from the northern and southern hemispheres are separated. Signal events are equally distributed in the northern and southern hemispheres, while backgrounds are stronger in the down-going southern hemisphere.

\begin{tabular}{|c|c|c|c|}
\hline Charge & $\begin{array}{l}\text { Signal Rate }\left(y r^{-1}\right) \\
\left(R_{s}\right)\end{array}$ & $\begin{array}{l}\text { Background Rate }\left(y r^{-1}\right) \\
\left(R_{b}\right)\end{array}$ & $S N R=R_{s} / R_{b}$ \\
\hline 6000 & $1.09(0.50 \mathrm{~N}+0.59 \mathrm{~S})$ & $3.73(0.67 N+3.06 \mathrm{~S})$ & $0.29(0.75 \mathrm{~N}, 0.19 \mathrm{~S})$ \\
\hline 6500 & $1.00(0.47 \mathrm{~N}+0.53 \mathrm{~S})$ & $2.81(0.58 \mathrm{~N}+2.23 \mathrm{~S})$ & $0.36(0.81 \mathrm{~N}, 0.24 \mathrm{~S})$ \\
\hline 7000 & $0.91(0.42 \mathrm{~N}+0.49 \mathrm{~S})$ & $1.16(0.49 \mathrm{~N}+0.67 \mathrm{~S})$ & $0.78(0.86 \mathrm{~N}, 0.73 \mathrm{~S})$ \\
\hline 7500 & $0.84(0.38 \mathrm{~N}+0.46 \mathrm{~S})$ & $0.92(0.41 \mathrm{~N}+0.51 \mathrm{~S})$ & $0.91(0.93 \mathrm{~N}, 0.90 \mathrm{~S})$ \\
\hline
\end{tabular}

\subsection{EHE alerts}

The extremely-high-energy (EHE) neutrino alert stream is based on an offline search for GZK, or cosmogenic, neutrinos that resulted in the discovery of the first observed PeV-scale neutrinos. 23 The analysis selection is simple and robust, making it a natural candidate to move into the online alert framework where computing resources are limited.

The offline diffuse EHE analysis targets neutrinos with energies of $\sim 10 \mathrm{PeV}-1 \mathrm{EeV}$, where the expected event rate in the most optimistic case is $\sim 1$ event per year. ${ }_{46}$ When moving this analysis into the realtime framework, the event selection was modified in order to increase the sensitivity to astrophysical neutrinos, specifically with neutrino energies in the $500 \mathrm{TeV}-10 \mathrm{PeV}$ range, targeting tracklike events, which have good angular resolution $\left(<1^{\circ}\right)$.

\subsubsection{EHE event selection and alerts}

The EHE alert selection requires a minimum deposited charge of $10^{3.6}$ photoelectrons (NPE) detected in DOMs in the detector volume as well as at least 300 DOMs registering a signal. The events are then fit with a track hypothesis and the fit quality parameter (charge weighted $\left.\chi^{2}\right)^{47}$ is required to be consistent with well reconstructed tracks.

Due to large background contamination from atmospheric muons originating in cosmic-ray air showers, there is an additional two-dimensional cut in the plane of detected zenith angle, $\cos (\vartheta), \operatorname{and} \log _{10}(\mathrm{NPE})$ :

- if $\cos (\vartheta) \leq 0.1$, then $\log _{10}(\mathrm{NPE})>3.6$

- if $\cos (\vartheta)>0.1$, then $\log _{10}(\mathrm{NPE})>3.6+2.99$

$\times \sqrt{1-\left(\frac{\cos \theta-0.93}{0.83}\right)^{2}}$ 
The two-dimensional selection was determined by optimizing for maximum signal retention assuming an astrophysical $\mathrm{E}^{-2}$ neutrino flux while tolerating some contamination from atmospheric backgrounds. This requirement is illustrated in Fig. 6 for the total estimated background from simulation (atmospheric muons and atmospheric muon neutrinos) and for a simulated astrophysical signal assuming

$\emptyset=1.0 \times 10^{-18}\left(\frac{E}{100 \mathrm{TeV}}\right)^{-2}\left[\mathrm{GeV}^{-1} \mathrm{~cm}^{-2} \mathrm{~s}^{-1} \mathrm{sr}^{-1}\right] .^{-1} \underline{\mathrm{Fig} .6}$ also illustrates the increased signal acceptance for the online alert selection compared to the offline diffuse analysis. The neutrino effective area for the online selection is calculated and is shown in Fig. 7 for the entire sky, as well as separately for the northern and southern hemispheres, and reflects an overall increase in sensitivity to events in the $\mathrm{TeV}$ and $\mathrm{PeV}$ range with respect to the offline diffuse analysis.
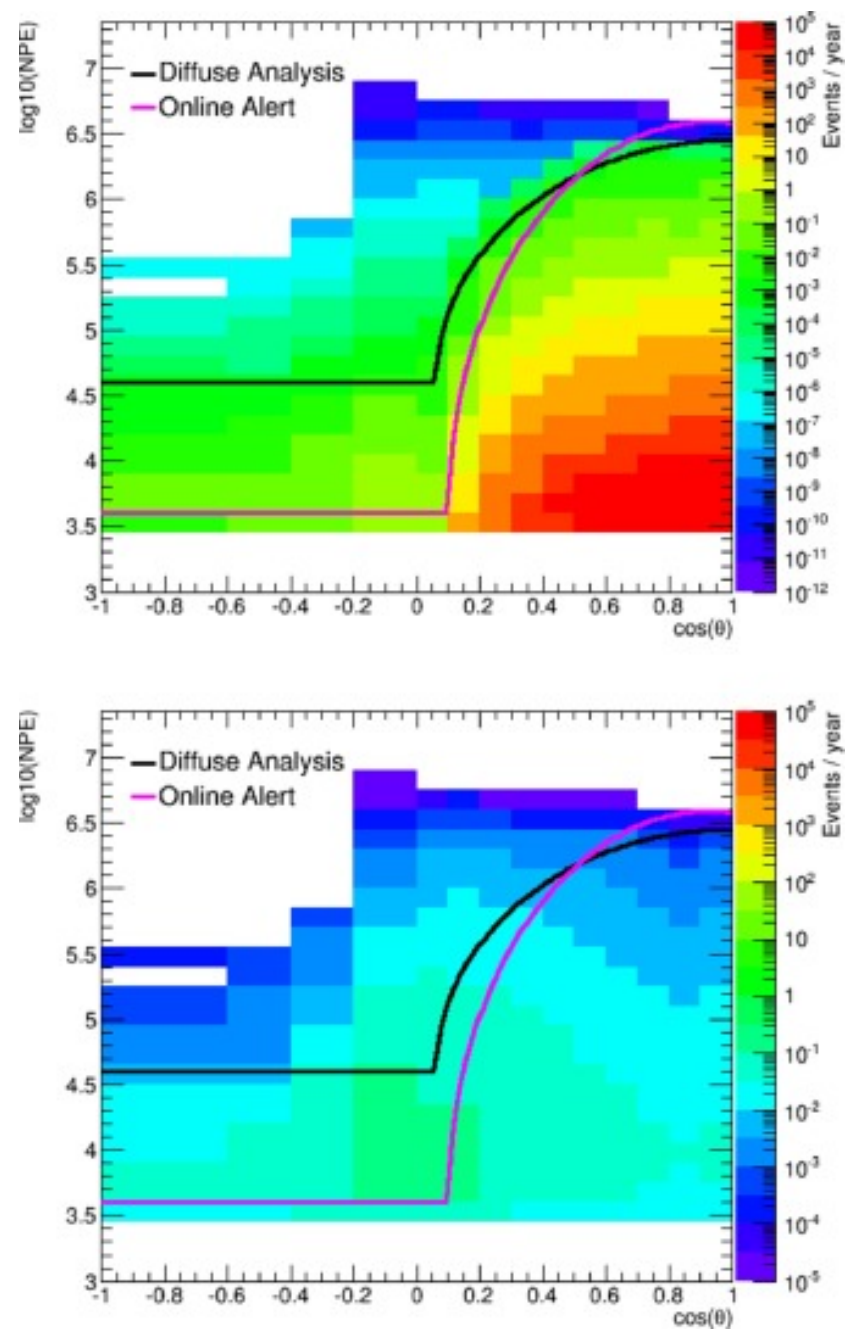

Fig. 6. The two-dimensional plane of $\cos (\theta)$ and $\log _{10}(N P E)$ for the summed background (atmospheric muons and atmospheric neutrinos) on the upper panel and signal (astrophysical $\mathrm{v}_{\mu}$ assuming an $E^{-2}$ spectrum) on the lower panel. Both the offline ultra-high-energy diffuse analysis (black ${ }^{24}$ ) and EHE Alert selection (magenta) are shown, with any event found above the line being selected. Few astrophysical signal events are expected for $\cos (\theta)<-0.3$ at large $\log _{10}(N P E)$ values $(>\sim 5.5)$ due to neutrino absorption in the Earth. 
The measured neutrino spectrum from contained event searches $\underline{45}$ and from through-going track searches ${ }^{2}$ return different spectral indices. Given these uncertainties, the expected event rate for signal is also estimated from simulated $v_{\mu}$ events weighted to a flux of $\emptyset=2.3 \times$ $10^{-18}\left(\frac{E}{100 \mathrm{TeV}}\right)^{-2.49}\left[\mathrm{GeV}^{-1} \mathrm{~cm}^{-2} \mathrm{~s}^{-1} \mathrm{sr}^{-1}\right]$ 出 is also calculated. The total number of alerts, classified by background type and signal assumption, is given in Table 2.

Table 2. Summary of the expected event alert rate for the EHE online alert stream for each sample per year, including expected contributions from backgrounds and 2 astrophysical neutrino spectra. The expected signal to noise ratio for this search is $\sim 2$.

\begin{tabular}{|l|l|}
\hline Sample & Events/year \\
\hline Atmospheric muon & 0.52 \\
\hline Conv. Atmos. $\mathbf{v}_{\boldsymbol{\mu}}$ & 1.20 \\
\hline Prompt Atmos. $\mathbf{v}_{\boldsymbol{\mu}}$ & 0.19 \\
\hline Total Backround & 1.91 \\
\hline Astro $\mathbf{v}_{\boldsymbol{\mu}}\left(\mathrm{E}^{-2}\right)$ & 4.09 \\
\hline Astro. $\mathbf{v}_{\mu}\left(\mathrm{E}^{-2.49}\right)$ & 2.48 \\
\hline
\end{tabular}

In addition to this prediction, the total alert rate has been validated using four years of archival IceCube data. The observed rate of 4.25 events per year is in agreement with background + signal hypothesis if the IceCube astrophysical diffuse global fit spectral fit results ${ }_{48}$ are assumed. Each event found in the archival data search has been visually inspected to confirm that they are, in fact, tracks.

A signalness parameter is calculated to provide a measure of how likely each event is to be of astrophysical origin relative to the total background rate. The signalness parameter is estimated from simulation by creating a two-dimensional probability map in the plane of $\cos (\vartheta)$ and $\log _{10}(\mathrm{NPE})$, following $P_{i, j}=N_{i, j}^{s i g} /\left(N_{i, j}^{s i g}+N_{i, j}^{b k g}\right)$, where i and $\mathrm{j}$ are the bins in the 2D plane, $\mathrm{N}^{\text {sig }}$ is the expected astrophysical $v_{\mu}$ signal (assuming an $\mathrm{E}^{-2}$ spectrum) in each bin, and $\mathrm{N}^{b k g}$ is the expected atmospheric muon and neutrino background in the bin. The resulting map can be seen in Fig. 8 . Bins are only filled if there is a minimum signal expectation from simulations of at least 10 events / year. The probability is capped at $95 \%$ due to limited statistics available in each bin. In the case that a data event falls within the white space where we do not have Monte Carlo coverage, the signalness value is set to -1 , and no alert is sent. 

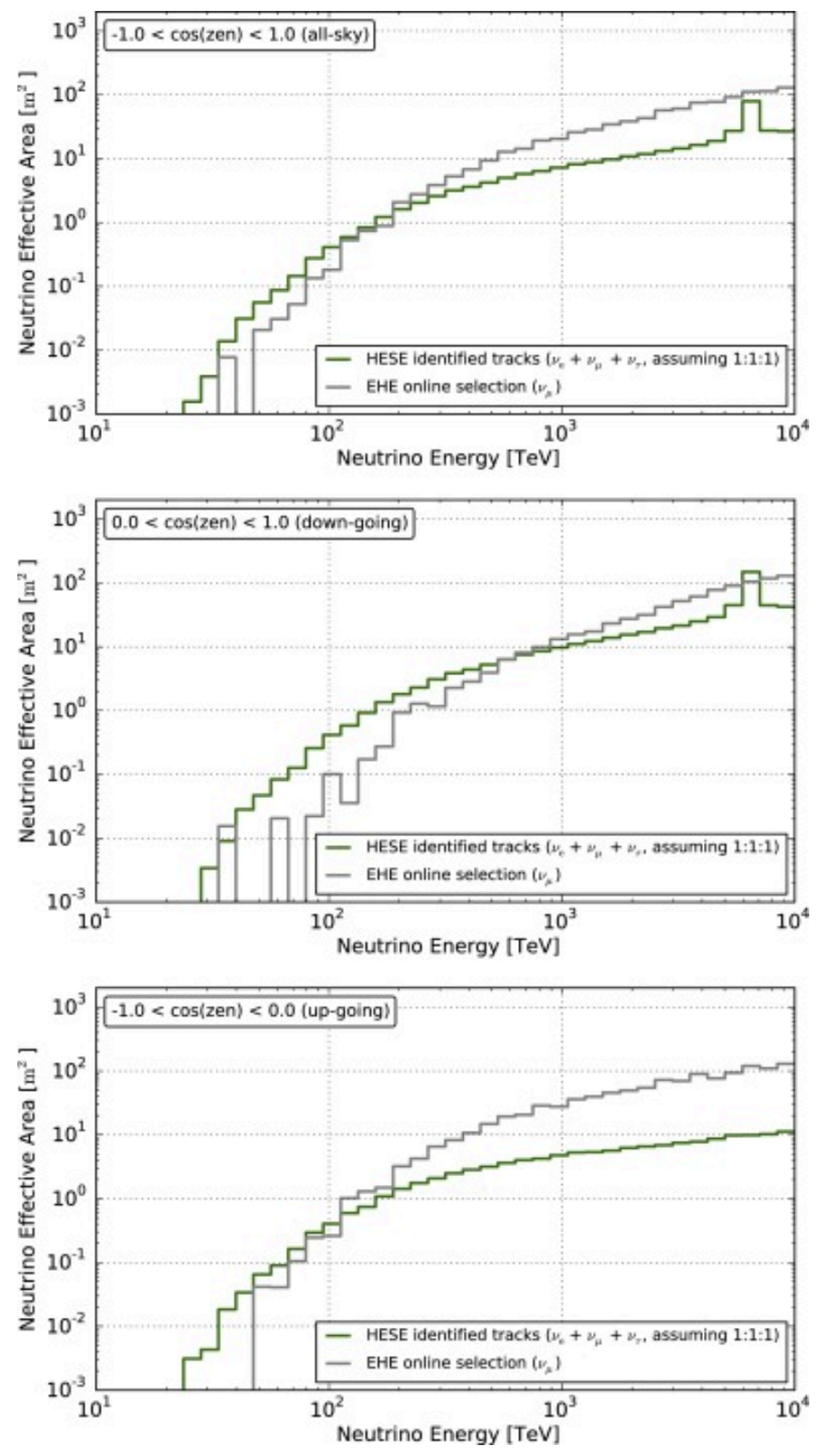

Fig. 7. The effective area as a function of neutrino energy for $v_{\mu}$ events for the online EHE signal diffuse neutrino selection and the for the HESE identified tracks (presented as the sum of the three per-flavor effective areas assuming 1:1:1 between neutrino flavors, and dominated by the $\mathrm{v}_{\mu}$ component). The top panel shows the all-sky effective area, while the down-going (southern sky) and up-going (northern sky) effective areas are shown in the middle and bottom panels, respectively. The EHE effective areas are increased relative to the published offline selection, $\underline{46}$ while the HESE effective areas shown here are just for track-like events and show reduced effective area relative to the published analysis. $\underline{12}$ 


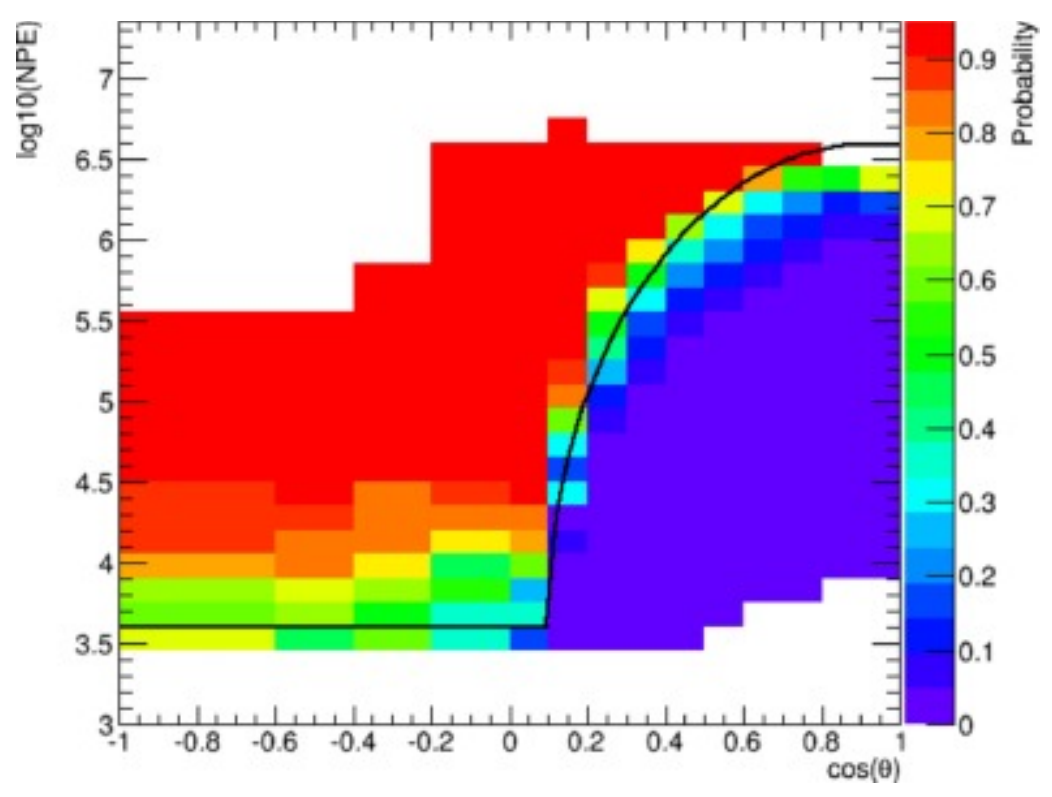

Fig. 8. The two-dimensional signalness map in the plane of $\cos (\vartheta)$ and $\log _{10}(N P E)$ derived from simulated events. Bins are only filled if a minimum threshold of signal events is met, and empty bins are indicated by white space.

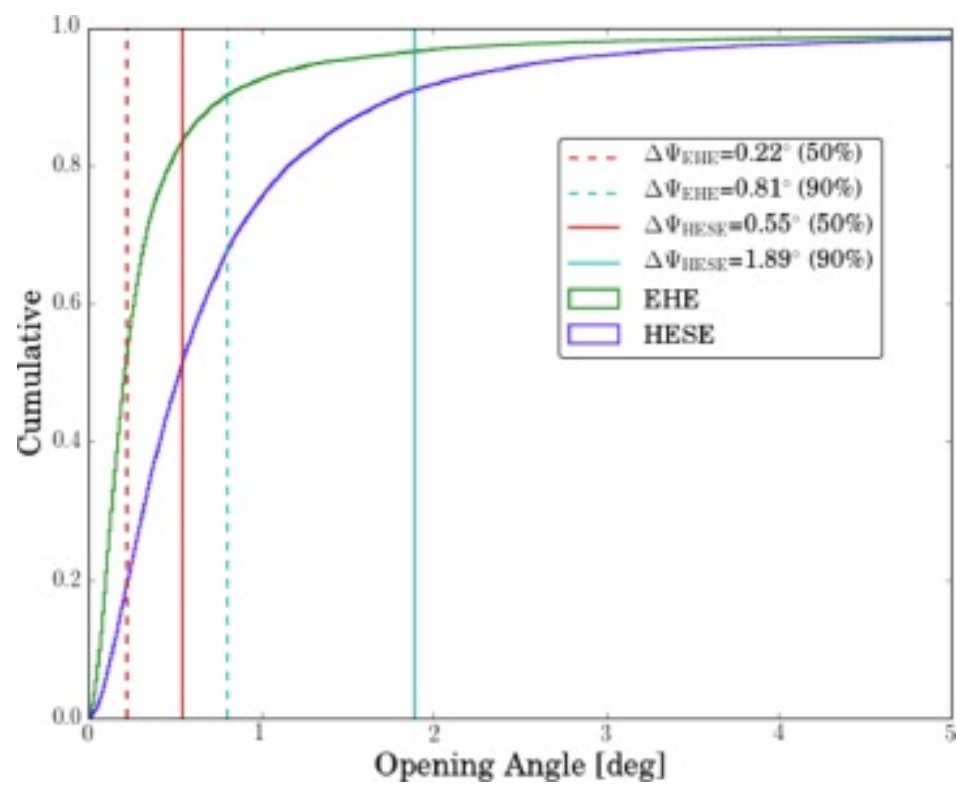

Fig. 9. The cumulative distribution for the opening angle showing the radius containing $50 \%$ and $90 \%$ of simulated events for HESE and EHE selected neutrino events. The largest variation in the HESE angular resolution comes from reconstructed track length. Here $80 \%$ the HESE track-like events with a reconstructed track length $>200 \mathrm{~m}$ are considered. The remaining 20\% are reported with an upper limit fixed median angular error of $1.6^{\circ}$ (8.9 $9^{\circ}$ for $90 \%$ containment). Systematic errors are not included.

Events passing the EHE alert selection will generate a public alert via the GCN network sent via AMON. These alerts will contain the best-fit source direction and angular uncertainty from the online reconstruction, date and time of the event, total measured charge, and measured signalness parameter. 
The angular resolution has been studied utilizing simulated $v_{\mu}$ events passing the EHE alert selection criteria by investigating the opening angle between the best available online fit to the observed muon track and the true neutrino direction. The median angular resolution is found to be $0.22^{\circ}$, as illustrated in Fig. 9 , and is nearly flat across the neutrino energy spectrum. As with the HESE alerts, after the initial alert generation, follow-up reconstructions of the EHE events by computer clusters in the northern hemisphere are immediately started, and revised directional coordinates and improved angular uncertainty are released within a few hours of the initial alert (see Section 2.3.2).

\section{Summary}

This paper provides a description of IceCube's realtime alert system, and the current active data alert streams. The computing infrastructure at both the South Pole facility as well as in the northern hemisphere allows IceCube to communicate, in real-time, the observation of high quality candidate neutrino singlets and multiplets. Within this framework, several analyses are currently implemented and sending alerts to our follow-up partners, as well as to the wider astronomical community through AMON. This system enables the existing analyses to be improved further and add new ones rapidly to respond to our evolving understanding of the astrophysical neutrino signal observed by IceCube.

With the establishment of the IceCube realtime alert system, the alerts generated by IceCube, and the potential discovery of transient astronomical sources in conjunction with them, the era of multimessenger time domain astronomy has arrived. High-energy neutrinos are a unique messenger, able to travel astronomical distances with negligible deflection or absorption, and clearly indicative of high energy hadrons in their sources. A clear multi-messenger detection of a source holds the potential to enrich our understanding of the most energetic cosmic phenomena, shed light on the mysterious origins of the highest energy cosmic rays, and provide a unique window into the cosmos.

\section{Acknowledgments}

We acknowledge the support from the following agencies: U.S. National Science Foundation-Office of Polar Programs, U.S. National Science Foundation- Physics Division, University of Wisconsin Alumni Research Foundation, the Grid Laboratory Of Wisconsin (GLOW) grid infrastructure at the University of Wisconsin - Madison, the Open Science Grid (OSG) grid infrastructure; U.S. Department of Energy, and National Energy Research Scientific Computing Center, the Louisiana Optical Network Initiative (LONI) grid computing resources; Natural Sciences and Engineering Research Council of Canada, WestGrid and Compute/Calcul Canada; Swedish Research Council, Swedish Polar Research Secretariat, Swedish National Infrastructure for Computing (SNIC), and Knut and Alice Wallenberg Foundation, Sweden; German Ministry for Education and Research (BMBF), Deutsche Forschungsgemeinschaft (DFG), Helmholtz Alliance for Astroparticle Physics (HAP), Research Department of Plasmas with Complex Interactions (Bochum), Germany; Fund for Scientific Research (FNRS-FWO), FWO Odysseus programme, Flanders Institute to encourage scientific and technological research in industry (IWT), Belgian Federal Science Policy Office (Belspo); University of Oxford, United Kingdom; Marsden Fund, New Zealand; Australian Research Council; Japan Society for Promotion of Science (JSPS); the Swiss National Science Foundation (SNSF), Switzerland; National Research Foundation of Korea (NRF); Villum Fonden, Danish National Research Foundation (DNRF), Denmark 


\section{References}

1. M.G. Aartsen, et al. Evidence for astrophysical muon neutrinos from the northern sky with IceCube. Phys. Rev. Lett., 115 (8) (2015), p. 081102, 10.1103/PhysRevLett.115.081102

2. M.G. Aartsen, et al. Observation and characterization of a cosmic muon neutrino flux from the northern hemisphere using six years of IceCube data. Astrophys. J., 833 (1) (2016), p. 3, 10.3847/0004-637X/833/1/3

3. K.A. Olive, et al. Review of particle physics. Chin. Phys., C38 (2014), p. 090001, 10.1088/16741137/38/9/090001

4. E. Waxman, J.N. Bahcall. High-energy neutrinos from cosmological gamma-ray burst fireballs. Phys. Rev. Lett., 78 (1997), pp. 2292-2295, 10.1103/PhysRevLett.78.2292

5. S. Razzaque, P. Meszaros, E. Waxman. High energy neutrinos from a slow jet model of core collapse supernovae. Mod. Phys. Lett., A20 (2005), pp. 2351-2368, 10.1142/S0217732305018414

6. D. Eichler. High-energy neutrino astronomy: a probe of galactic nuclei? Astrophys. J., 232 (1979), pp. 106-112, 10.1086/157269

7. A. Achterberg, et al. First year performance of the IceCube neutrino telescope. Astropart. Phys., 26 (2006), pp. 155-173, 10.1016/i.astropartphys.2006.06.007

8. M.G. Aartsen, et al. The IceCube neutrino observatory: instrumentation and online systems. JINST, 12 (2017), p. P03012

9. R. Abbasi, et al. The design and performance of IceCube deepcore. Astropart. Phys., 35 (2012), pp. 615-624, 10.1016/j.astropartphys.2012.01.004

10. M.G. Aartsen, et al. Measurement of South Pole ice transparency with the IceCube LED calibration system. Nucl. Instrum. Methods, A711 (2013), pp. 73-89, 10.1016/i.nima.2013.01.054

11. M.G. Aartsen, et al. Energy reconstruction methods in the IceCube neutrino telescope. JINST, 9 (2014), p. P03009, 10.1088/1748-0221/9/03/P03009

12. M.G. Aartsen, et al. Observation of high-energy astrophysical neutrinos in three years of IceCube data. Phys. Rev. Lett., 113 (2014), p. 101101, 10.1103/PhysRevLett.113.101101

13. T. Kintscher. Results and prospects of IceCubes real time alert capabilities. J. Phys. Conf. Ser., 718 (6) (2016), p. 062029, 10.1088/1742-6596/718/6/062029

14. R. Abbasi, et al. .Searching for soft relativistic jets in core-collapse supernovae with the IceCube optical follow-up program. Astron. Astrophys., 539 (2012), p. A60, 10.1051/0004$\underline{6361 / 201118071}$

15. M.G. Aartsen, et al. Detection of a type Iln supernova in optical follow-up observations of IceCube neutrino events. Astrophys. J., 811 (1) (2015), p. 52, 10.1088/0004-637X/811/1/52

16. P.A. Evans, et al. Swift follow-up of IceCube triggers, and implications for the advanced-LIGO era. Mon. Not. Roy. Astron. Soc., 448 (3) (2015), pp. 2210-2223, 10.1093/mnras/stv136

17. M.G. Aartsen, et al. Very high-energy gamma-ray follow-up program using neutrino triggers from IceCube. JINST, 11 (2016), p. P11009, 10.1088/1748-0221/11/11/P11009

18. M. Santander, The dawn of multi-messenger astronomy(2016).

19. E. Blaufuss, GCN circular 19363. [link]. URL http://gcn.gsfc.nasa.gov/gcn3/19363.gcn3.

20. R. Abbasi, et al. The IceCube data acquisition system: signal capture, digitization, and timestamping. Nucl. Instrum. Methods, A601 (2009), pp. 294-316, 10.1016/j.nima.2009.01.001 
21. R. Abbasi, et al. Calibration and characterization of the IceCube photomultiplier tube. Nucl. Instrum. Methods, A618 (2010), pp. 139-152, 10.1016/j.nima.2010.03.102

22. M.G. Aartsen, et al. Searches for extended and point-like neutrino sources with four years of IceCube data. Astrophys. J., 796 (2) (2014), p. 109, 10.1088/0004-637X/796/2/109

23. M.G. Aartsen, et al. First observation of PeV-energy neutrinos with IceCube. Phys. Rev. Lett., 111 (2013), p. 021103, 10.1103/PhysRevLett.111.021103

24. M.G. Aartsen, et al. Probing the origin of cosmic rays with extremely high energy neutrinos using the IceCube observatory. Phys. Rev., D88 (2013), p. 112008, 10.1103/PhysRevD.88.112008

25. M.G. Aartsen, et al. Evidence for high-energy extraterrestrial neutrinos at the IceCube detector. Science, 342 (2013), p. 1242856, 10.1126/science.1242856

26. R. Seaman, et al., IVOA recommendation: sky event reporting metadata version 2.0 (2011).

27. M.W.E. Smith, et al. The astrophysical multimessenger observatory network (AMON). Astropart. Phys., 45 (2013), pp. 56-70, 10.1016/j.astropartphys.2013.03.003

28. Gamma-ray coordinates network. http://gcn.gsfc.nasa.gov

29. M.G. Aartsen, et al. All-sky search for time-integrated neutrino emission from astrophysical sources with 7 years of IceCube data Astrophys. J., 835 (2) (2017), p. 151, 10.3847/15384357/835/2/151

30. Y. Freund, R.E. Schapire. A decision-theoretic generalization of on-line learning and an application to boosting. J. Comput. Syst. Sci., 55 (1) (1997), pp. 119-139, 10.1006/jcss.1997.1504

31. I. Tamborra, S. Ando. Inspecting the supernova gamma-ray-burst connection with high-energy neutrinos. Phys. Rev., D93 (5) (2016), p. 053010, 10.1103/PhysRevD.93.053010

32. N. Senno, K. Murase, P. Meszaros. Choked jets and low-luminosity gamma-ray bursts as hidden neutrino sources. Phys. Rev., D93 (8) (2016), p. 083003, 10.1103/PhysRevD.93.083003

33. C.W. Akerlof, et al. The ROTSE-III robotic telescope system. Publ. Astron. Soc. Pac., 115 (2003), pp. 132-140, 10.1086/345490

34. N.M. Law, et al. The Palomar transient factory: system overview, performance and first results. Publ. Astron. Soc. Pac., 121 (2009), p. 1395, 10.1086/648598

35. D.N. Burrows, et al. The swift X-ray telescope. Space Sci. Rev., 120 (2005), p. 165, 10.1007/s11214-005-5097-2

36. V. Lipunov, et al. Master robotic net. Adv. Astron., 2010 (2010), p. 349171, 10.1155/2010/349171

37. ASAS-SN telescope. http://www.astronomy.ohio-state.edu/ assassin

38. Pickles, et al. LCOGT network observatory operations. Proc. SPIE, 9149 (2014), p. 914912, $10.1117 / 12.2055215$

39. P.L. Nolan, et al. Fermi large area telescope second source catalog. Astrophys. J. Suppl., 199 (2012), p. 31, 10.1088/0067-0049/199/2/31

40. J. Aleksić, et al. The major upgrade of the MAGIC telescopes, part II: a performance study using observations of the Crab Nebula. Astropart. Phys., 72 (2016), pp. 76-94, 10.1016/i.astropartphys.2015.02.005

41. N. Park. Performance of the VERITAS experiment. PoS, ICRC2015 (2016), p. 771 
42. J. Braun, J. Dumm, F. De Palma, C. Finley, A. Karle, T. Montaruli. Methods for point source analysis in high energy neutrino telescopes. Astropart. Phys., 29 (2008), pp. 299-305, 10.1016/i.astropartphys.2008.02.007

43. D. Gora, E. Bernardini, A.H. Cruz Silva. A method for untriggered time-dependent searches for multiple flares from neutrino point sources. Astropart. Phys., 35 (2011), pp. 201-210, 10.1016/j.astropartphys.2011.07.007

44. F. Schüssler, et al. The H.E.S.S. multi-messenger program. PoS, ICRC2015 (2016)

45. C. Kopper, W. Giang, N. Kurahashi. Observation of astrophysical neutrinos in four years of IceCube data. PoS, ICRC2015 (2016), p. 1081

46. M.G. Aartsen, et al. Constraints on ultra-high-energy cosmic ray sources from a search for neutrinos above 10 PeV with IceCube. Phys. Rev. Lett., 117 (24) (2016), p. 241101, 10.1103/PhysRevLett.117.241101

47. M.G. Aartsen, et al. Improvement in fast particle track reconstruction with robust statistics. Nucl. Instrum. Methods, A736 (2014), pp. 143-149, 10.1016/j.nima.2013.10.074

48. L. Mohrmann. Combined analysis of the high-energy cosmic neutrino flux at the IceCube detector. PoS, ICRC2015 (2016), p. 1066 